\title{
NUTRITIONAL STATUS DIFFERENTIALLY AFFECT YIELD AND MUST COMPOSITION OF HYBRIDS AND $V$. VINIFERA VARIETIES ESTABLISHED UNDER COLD CLIMATE CONDITIONS
}

\section{EFEITO DO ESTADO NUTRICIONAL NO RENDIMENTO E NA COMPOSIÇÃO DO MOSTO DE VARIEDADES DE HÍBRIDOS E DE $V$. VINIFERA INSTALADAS EM CLIMAS FRIOS}

\author{
Francisco Diez-Zamudio ${ }^{1}{ }^{1}$, Rodrigo Laytte ${ }^{2}$, Cecilia Grallert ${ }^{2}$ and Gastón Gutiérrez-Gamboa ${ }^{3, *}$ \\ ${ }^{1}$ Perennia Food and Agriculture Inc. 32 Main street, Kentville, Nova Scotia, B4N 1J5, Canada. \\ ${ }^{2}$ LG Terroir and Wine Consulting. 12 Avenue des Mésanges, 33320 Le Taillan Medoc, France. \\ ${ }^{3}$ Escuela de Agronomía, Facultad de Ciencias, Universidad Mayor, Camino La Pirámide 5750, Huechuraba 8580000, Chile.
}

* Corresponding authors: Tel.: +56 979942130,+1 707 5929315. e-mail: gaston.gutierrez@umayor.cl, fdiez@perennia.ca

(Received 03.02.2021. Accepted 06.07.2021)

\section{SUMMARY}

Nova Scotia is characterized by cold climate and acidic soils and high organic matter for viticultural development. There is little scientific information available about the nutritional management of grapevine varieties cultivated under cold climate conditions even in Nova Scotia. This study carried out in three seasons aimed to find correlations between tissue nutrients with yield and must composition in order to provide better nutritional management for a given variety. The yield of "L'Acadie" variety was correlated with B, K and Mg. In this variety, $\mathrm{Ca}$ to $\mathrm{Mg}$ ratio could be an interesting indicator of yield. The yield of "New York Muscat" variety was correlated with Zn, while N, P and K was related to bunch weight and weight of berries. Interspecific hybrids presented lower accumulation of B in their tissues than $V$. vinifera varieties. $\mathrm{Zn}$ and Fe were found as determinant micronutrients in "Chardonnay" variety. "Riesling" variety accumulated high levels of K in their tissues even over the optimal values recommended for grapevines. $\mathrm{K}$ to $\mathrm{Ca}$ ratio could be an important indicator of yield in "Pinot Noir" variety. A strong correlation between the petiole and blade analysis was found in macro and micro-nutrients. These results may contribute to improve the nutritional management of grapevines grown under cold climate conditions, mostly in Nova Scotia.

\section{RESUMO}

A Nova Escócia é caracterizada por um clima frio e solos ácidos com teores elevados de matéria orgânica, condições onde ocorre a cultura da vinha. Existe pouca informação científica disponível sobre a nutrição e fertilização da vinha cultivada em climas frios. Este estudo, realizado em três vindimas, teve como objetivo encontrar correlações entre os nutrientes dos pecíolos e limbos com a produção e a composição do mosto, a fim de proporcionar uma melhor gestão nutricional para algumas variedades. A produção da variedade "L'Acadie" esteve associada aos teores foliares de $\mathrm{B}, \mathrm{K}$ e $\mathrm{Mg}$. Nesta variedade, a relação $\mathrm{Ca} / \mathrm{Mg}$ pode ser um indicador interessante da sua produtividade. A produção da variedade "New York Muscat" demonstrou estar relacionada com o teor foliar de Zn, enquanto os teores de $\mathrm{N}, \mathrm{P}$ e K foram associados ao peso do cacho e ao peso dos bagos. Híbridos interespecíficos apresentaram menor acumulação de B nos seus tecidos em comparação com as variedades de $V$. vinifera. O Zn e o Fe foram identificados como micronutrientes determinantes em alguns parâmetros de qualidade do mosto na variedade "Chardonnay". A variedade "Riesling" acumulou altos níveis de K nos seus tecidos, mesmo acima dos valores ideais recomendados para videiras. A razão K/Ca pode ser um indicador importante da produção na variedade "Pinot Noir". Encontrou-se uma forte correlação entre a composição em macro e micronutrientes do pecíolo e do limbo. Os resultados obtidos podem contribuir para uma melhor nutrição das vinhas cultivadas em climas frios, principalmente na Nova Escócia.

Keywords: cold climate, Nova Scotia, hybrids, "L’Acadie", "New York Muscat".

Palavras-chave: clima frio, Nova Scotia, híbridos, "L'Acadie", "New York Muscat".

\section{INTRODUCTION}

In recent decades, a small yet growing wine industry has developed in Nova Scotia (Canada) close to the northern climatic limit for viticulture (Point et al., 2012). By 2015 there were 70 grape growers and nearly two dozen wineries in the province accounting 256 ha of vineyards in production (Withers, 2015). Data from 2019 shows an increase of almost $50 \%$ of acreage with over 400 hectares under production through Nova Scotia (Grallert and Laytte, 2018).

89

This is an Open Access article distributed under the terms of the Creative Commons Attribution License (https://creativecommons.org/licenses/by/4.0), which permits unrestricted use, distribution, and reproduction in any medium, provided the original work is properly cited 
There are four main wine-growing regions in Nova Scotia that are influenced by the warming effects of the Bay of Fundy and Atlantic Ocean as Malagash Peninsula, Annapolis Valley, Gaspereau Valley and the South Shore (Cameron et al., 2012). Cold winters and a short growing season pose paramount challenges to growers and winemakers in the provinces of Nova Scotia (Shaw, 2007). Given Nova Scotia's climate conditions, most of the vineyard surface is planted with hearty French hybrid varieties, mainly 'L'Acadie", but growers are having a recent success in the warmest areas of the region with Vitis vinifera varieties such as "Chardonnay", "Riesling" and "Pinot Noir" (Fisher and Jamieson, 2000; Naugler et al., 2007; Point et al., 2012; Pedneault and Provost, 2016). However, large-scale production of the cold-sensitive $V$. vinifera varieties demanded by consumers depends greatly on their surviving the extremes of winter and reaching maturity in the short, cool growing season (Shaw, 2007). The white hybrids cultivated in Nova Scotia include "L'Acadie", "New York Muscat", "Seyval Blanc", and "Vidal Blanc", while the white $V$. vinifera include "Chardonnay", "Riesling", "Pinot Gris", and "Sauvignon Blanc" varieties (Fisher and Jamieson, 2000; Cameron et al., 2012; Pedneault and Provost, 2016). Red hybrids growing in Nova Scotia are "Baco Noir", "Léon Millot", "Lucie Kuhlmann", and "Maréchal Foch", whereas the main red $V$. vinifera variety cultivated is "Pinot Noir" (Cameron et al., 2012; Pedneault and Provost, 2016). Nova Scotia has nearly perfect climatic conditions for making world class ice-wines and sparkling wines (Naugler et al., 2007; Cameron et al., 2012).

Nova Scotia is characterized to having soils with low $\mathrm{pH}$ and high organic matter for viticulture which conditioned the absorption of nutrients from the soil by the roots of the vines. Nova Scotia's soils are podzolic soils according to the Canadian system of soil classification (Sangster, 2018). These soils are distinguished by their high acidity in the A horizon, which results in formation of a bleached layer (the Ae horizon) and deposition of iron and aluminum in the B (Sangster, 2018). Contrary to this, in a terroir study, Grallert and Laytte (2018) reported that none of the vineyard soils are Podzosol and most of the analyzed soils are principally sandy soils, which display a low quantity of coarse elements, important levels of organic matter, a low to high soil acidity and a generalized $\mathrm{K}$ deficiency. Additionally, a medium to high compaction could be observed in many soil profiles (Grallert and Laytte, 2018). For good agricultural development, lime is necessary to adjust the $\mathrm{pH}$ of the naturally acidic soils in Nova Scotia (Bishop et al., 1969; Jensen et al., 2004; Sangster, 2018). Low $\mathrm{pH}$ results in lower rates of $\mathrm{N}$ mineralization, a process dependent on active, viable microbial populations in the soil (Neina, 2019). These conditions result in high concentrations of aluminum $\left(\mathrm{Al}^{3+}\right)$, ammonium $\left(\mathrm{NH}_{4}^{+}\right)$, and hydrogen ions $\left(\mathrm{H}_{3} \mathrm{O}^{+}\right)$what tends to inhibit $\mathrm{Mg}$ uptake from soils (Keller, 2020). Studies suggest that an increase in soil $\mathrm{pH}$ resulted in an increase in $\mathrm{Mo}$ and $\mathrm{Cu}$ content of plant tissue while $\mathrm{B}, \mathrm{Mn}$, and $\mathrm{Zn}$ decreased (Gupta et al., 1971; Fageria and Nascente 2014; Rutkowska et al., 2017). Liming to a pH of 5.6 and above reduced the $\mathrm{Mn}$ and $\mathrm{Zn}$ availability of soils (Gupta et al., 1971; Rengel, 2015). Gupta et al. (1971) showed that the B available was low at $\mathrm{pH}$ values greater than 6.1 in Nova Scotia soils and $\mathrm{N}$ and $\mathrm{Zn}$ additions may be required on the high $\mathrm{pH}$ soils of Eastern Canada in the future. In a recent terroir study was shown that interspecific hybrids cultivated in Annapolis Valley contained lower B in petioles and $\mathrm{N}$ in berries than the $V$. vinifera varieties growing in the same valley (Grallert and Laytte 2018; non-published data). In this way, the grapevine varieties established in Nova Scotia could have a different dynamic of nutrient absorption under similar edaphoclimatic conditions. To our knowledge, this is one of the first report showing evidence of nutritional management both hybrids and $V$. vinifera varieties growing in Nova Scotia (Messiga et al., 2016). Therefore, the aim of this study was to establish correlations between tissue nutrients and their ratios with yield and must physico-chemical composition in order to obtain more knowledge about the nutrient elements needs of the grapevine varieties cultivated in Nova Scotia. A second goal was proposed to evaluate the effects of variety, season and their interaction on blade and petiole nutrient content determined at bloom and véraison.

\section{MATERIALS AND METHODS}

\section{Study site and plant material}

The research was conducted across the Annapolis Valley, Nova Scotia, Canada $\left(45^{\circ} 00^{\prime} \mathrm{N} 64^{\circ} 55^{\prime} \mathrm{W}\right)$, over 2017, 2018 and 2019 seasons. Five different vineyards, which were located at a close distance from each other, were selected for the present study (Table 1). In two of them, vineyards were established with interspecific hybrid varieties, such as "L'Acadie" ("Cascade" × "Seyve-Villard 14-287") and "New York Muscat" ("Muscat Hamburg" $\times$ "Ontario"), while in the rest of the sites, the vineyards were planted with Vitis vinifera varieties, such as "Chardonnay", "Riesling" and "Pinot Noir". In each vineyard, three replications of 30 grapevines were randomly arranged. The selection of the vineyards followed a similar criterion: adult non-irrigated grapevines, trained to vertical shoot position system, growing in good phytosanitary conditions and with an active leaf surface area during the short growing season.

In the first season it was applied 2,000 kg per hectare (ha) of lime as dolomitic on every vineyard. In the second and third seasons, the applications diminished to 500 to $1,000 \mathrm{~kg}$ per ha in which was applied calcitic lime. At the beginning of the season 
of the third season, $50 \mathrm{~kg}$ of $\mathrm{P}_{2} \mathrm{O}_{5}$ per ha and $90 \mathrm{~kg}$ of $\mathrm{K}_{2} \mathrm{O}$ per ha were applied to the soil. In addition, $1 \mathrm{~kg}$ of $\mathrm{N}$ per ha, $15 \mathrm{~L}$ of seaweed per ha and $100 \mathrm{~g}$ per ha of chelate $\mathrm{Fe}$ was applied at the beginning of the second and third season as foliar application. Besides, in these seasons, from bloom until veraison, $2 \mathrm{~kg}$ of $\mathrm{K}_{2} 0$ per ha, $2 \mathrm{~kg}$ of $\mathrm{Mg}$ per ha, and $4 \mathrm{~kg}$ of $\mathrm{CaO}$ per ha were foliarly applied.

In 2017, yield per vine in "L'Acadie", "New York Muscat", "Chardonnay", "Riesling" and "Pinot
Noir" was $1.43 \pm 0.34, \quad 1.75 \pm 0.66, \quad 2.77 \pm 0.28$, $3.55 \pm 0.55$ and $1.82 \pm 0.64 \mathrm{~kg}$, respectively. In 2018 , yield per vine in "L'Acadie" and "Riesling" was $0.28 \pm 0.09$ and $1.63 \pm 0.45 \mathrm{~kg}$, respectively. In 2019, yield per vine in "L'Acadie", "New York Muscat", "Chardonnay", "Riesling" and "Pinot Noir" was $1.45 \pm 0.39,1.40 \pm 0.25,2.00 \pm 0.02,1.21 \pm 0.58$ and $\sim 1.70 \mathrm{~kg}$, respectively.

Table I Study site and plant material information

\begin{tabular}{|c|c|c|c|c|c|c|}
\hline \multirow[b]{2}{*}{ Variety } & \multirow[b]{2}{*}{ Species } & \multirow{2}{*}{$\begin{array}{l}\text { Season of } \\
\text { planting }\end{array}$} & \multicolumn{2}{|c|}{ Plantation distance (m) } & \multicolumn{2}{|c|}{ Plant characteristics } \\
\hline & & & $\begin{array}{c}\text { Between } \\
\text { rows }\end{array}$ & $\begin{array}{c}\text { Between } \\
\text { plants }\end{array}$ & Clone & Rootstock \\
\hline "L'Acadie" & Vitis interspecific crossing & 2005 & 3.1 & 0.9 & Unknown & Own rooted \\
\hline "New & & & & & & \\
\hline Muscat" & Vitis interspecific crossing & 1997 & 3.0 & 1.2 & Unknown & Own rooted \\
\hline "Chardonnay" & $V$. vinifera L., ssp. vinifera & 2003-2006 & 3.1 & 1.1 & 76 & 3309 Couderc \\
\hline "Riesling" & $V$. vinifera L., ssp. vinifera & 2013-2015 & 2.6 & 1.4 & 21B & 3309 Couderc \\
\hline "Pinot Noir" & $V$. vinifera L., ssp. vinifera & 2003-2006 & 3.1 & 1.1 & 115 & 3309 Couderc \\
\hline
\end{tabular}

\section{Climatic conditions and soil characteristics}

Climatic data was recorded using an automatically weather station (AWS) located close to each vineyard. Some bioclimatic indices, such as Winkler Index, Huglin's Heliothermal Index (HI), Growing Season Temperature (GST) and Cool Night Index
(CI), were calculated according to the stated by some researchers (Winkler, 1974; Huglin, 1978; Tonietto and Carbonneau, 2004; Jones, 2005). Free frost period, precipitations and chilling hours of each season were also recorded by the AWS as shown in Table 2.

Table II Climatic information and bioclimatic indices calculated each season under study

\begin{tabular}{|c|c|c|c|c|c|c|c|c|c|}
\hline \multirow[b]{2}{*}{ Season } & \multirow[b]{2}{*}{ WI } & \multicolumn{3}{|c|}{ Bioclimatic indices } & \multicolumn{3}{|c|}{ Free Frost Period } & \multirow[b]{2}{*}{$\begin{array}{c}\text { Precipitation } \\
\text { mm }\end{array}$} & \multirow[b]{2}{*}{$\begin{array}{c}\text { Chilling } \\
\text { hours }\end{array}$} \\
\hline & & HI & GST $\left({ }^{\circ} \mathrm{C}\right)$ & $\mathrm{CI}\left({ }^{\circ} \mathrm{C}\right)$ & Spring & Fall & $\begin{array}{c}\text { Free Frost } \\
\text { Days }\end{array}$ & & \\
\hline 2017 & 1169 & 1579 & 14.7 & 9.8 & 22-Apr-17 & 10-Nov-17 & 202 & 683 & 1399.8 \\
\hline 2018 & 1143 & 1535 & 14.2 & 7.2 & 5-Jun-18 & 24-Sep-18 & 111 & 898 & 1338.4 \\
\hline 2019 & 960 & 1515 & 12.5 & 7.7 & 4-May-19 & 25-Oct-19 & 174 & 633 & 1124.2 \\
\hline
\end{tabular}

${ }^{1}$ Footnote: WI: Winkler Index (Winkler, 1974); HI: Heliothermal Index (Huglin, 1978); GST: Growing Season Temperature (Jones, 2005); CI: Cool Night Index (Tonietto and Carbonneau, 2004).

Soil samples were collected from within the vine rows from the fourth vine to avoid the field edge effect. Subsequently, a sample was collected between vines within rows to a depth of approximately $30 \mathrm{~cm}$ of soil. Before this, in each vineyard it was performed a test pit to determine the physicochemical parameters of soils (Table 3). Soil testing was performed by Aurea Agroscience (Blanquefort, France). 
Determination of yield, yield components and must parameters

Bunch weight and weight of 200 berries were measured using an analytical balance (Adam CBK 8a, Oxford, UK). The productivity by grapevine was calculated as the sum of bunches weighted and collected by plant at harvest.

Table II Soil nutritional parameters obtained in the first $30 \mathrm{~cm}$ of soil

\begin{tabular}{|c|c|c|c|c|c|c|c|c|c|c|c|c|}
\hline Variety & pH & $\begin{array}{l}\text { OM* } \\
(\mathrm{g} / \mathrm{kg})\end{array}$ & Soil texture & $\begin{array}{c}\mathrm{P}_{2} \mathrm{O}_{5} \\
(\mathrm{mg} / \mathrm{kg})\end{array}$ & $\begin{array}{c}\mathrm{K}_{2} \mathrm{O} \\
(\mathrm{mg} / \mathrm{kg})\end{array}$ & $\begin{array}{c}\text { MgO } \\
\text { (mg/kg) }\end{array}$ & $\begin{array}{c}\mathrm{CaO} \\
(\mathrm{mg} / \mathrm{kg})\end{array}$ & $\begin{array}{c}\text { B } \\
(\mathrm{mg} / \mathrm{kg})\end{array}$ & $\begin{array}{c}\text { Mn } \\
\text { (mg/kg) }\end{array}$ & $\begin{array}{c}\mathrm{Cu} \\
(\mathrm{mg} / \mathrm{kg})\end{array}$ & $\begin{array}{c}\text { Fe } \\
(\mathrm{mg} / \mathrm{kg})\end{array}$ & $\begin{array}{c}\mathrm{Zn} \\
(\mathrm{mg} / \mathrm{kg})\end{array}$ \\
\hline "L'Acadie" & 5.9 & 50 & Sandy & 93 & 53 & 321 & 2140 & 0.22 & 3.52 & 1.15 & 129.88 & 1.43 \\
\hline "New York Muscat" & 6.8 & 28 & Sandy loam & 30 & 128 & 328 & 1550 & 0.47 & 2.81 & $<0.50$ & 119.67 & 3.98 \\
\hline "Chardonnay" & 6.4 & 30 & Sandy loam & 40 & 72 & 416 & 2020 & 0.29 & 2.03 & $<0.50$ & 150.28 & 1.59 \\
\hline "Riesling" & 6 & 35 & $\begin{array}{c}\text { Sandy clay } \\
\text { loam }\end{array}$ & 149 & 71 & 414 & 2120 & 0.51 & 2.98 & 0.95 & 360.49 & 2.8 \\
\hline "Pinot Noir" & 6.7 & 33 & $\begin{array}{c}\text { Sandy clay } \\
\text { loam }\end{array}$ & 70 & 130 & 501 & 2820 & 0.42 & 3.35 & $<0.50$ & 199.64 & 2.73 \\
\hline
\end{tabular}

*OM: Organic matter

The grapes were harvested from each replicate within the experimental vineyards, when the berries reached the following parameters: content of soluble solids of approximately $15-19{ }^{\circ}$ Brix and $\mathrm{pH}$ level between 2.8 and 3.2. Subsequently, the bunches were destemmed, and the grapes were crushed, and soluble solids, titratable acidity, $\mathrm{pH}$ and malic acid in musts were determined by the OIV methods (OIV, 2003, 2009). For the analysis of yeast assimilable nitrogen (YAN) content of musts, the samples were centrifuged with an Eppendorf Centrifuge model 5702R (Mississauga, Canada) at $3000 \mathrm{x}$ g for $5 \mathrm{~min}$. Then, the supernatants were analyzed by the Fourier transform infrared (FT-IR) analyzer using the FOSS WineScan ${ }^{\mathrm{TM}} \mathrm{SO}_{2}$ model, according to the method proposed by Skoutelas et al. (2011).

\section{Blade and petiole nutrient content}

Leaf petioles located at opposite side to the bunches and blades were collected at bloom and véraison stages of each studied season. Sixty to eighty petioles and blades per treatment and per replicates were collected. Macro (N, P, K, Ca and Mg) and micro (B, $\mathrm{Fe}$ and $\mathrm{Zn}$ ) nutrient content were analyzed. The ratios of $\mathrm{Ca}$ to $\mathrm{Mg}, \mathrm{K}$ to $\mathrm{Ca}, \mathrm{K}$ to $\mathrm{Mg}$ and the sum of $\mathrm{Ca}$ and $\mathrm{Mg}$ to $\mathrm{K}$ were also calculated for blades and petioles since they are used as indexes of grapevine nutritional status (Muthukrishnan and Srinivasan 1974; Assimakopoulou and Tsougrianis 2012). The combustion method (Leco CN828, Leco Instruments, USA) was used to analyze $\mathrm{N}$ content of blades and leaf petioles (AOAC International, 2006). Minerals were analyzed by Inductively Coupled Plasma Optical Emission Spectroscopy (Varian 725 ICP-OES, Agilent Technologies, USA), based on the method of AOAC International (2005). Macronutrient concentration was expressed in percentage (w/w), while micronutrients were expressed in $\mathrm{mg} / \mathrm{kg}$.

\section{Statistical analysis}

Linear correlations (r) were carried out between macro and micronutrient contents of blades and petioles analyzed in bloom and véraison with yield, yield components and must quality attributes by multivariate descriptive analysis using Statgraphics Centurion XVI.I statistical package (Figures 1 - 4). Petiole and blade nutrient content was subjected to a multifactorial analysis (MANOVA) using Statgraphics Centurion XVI.I statistical package. Non-linear regressions between petiole and blade content in samples collected at bloom and véraison were established for each analyzed nutrient (Figure $5)$. The coefficient of determination $\left(r^{2}\right)$ was used to evaluate how well the regression line represents the data.

\section{RESULTS AND DISCUSSION}

\section{Linear correlations in " $L$ 'Acadie"}

Linear correlations between nutrient status and yield, yield components and must parameters in "L'Acadie" is shown in Figures 1 and 2. As expected, $\mathrm{N}$ was positively correlated with yeast assimilable nitrogen in most of the samples. $\mathrm{N}$ analysed at véraison was positively correlated with malic acid, while B determined in this phenological stage does it inversely. $\mathrm{N}$ content measured at véraison was negatively correlated with soluble solids. In addition, $\mathrm{Ca}$ analysed in blades at véraison, and $\mathrm{P}$ petiole content measured in bloom and véraison were positively correlated with soluble solids. Regarding variables, bunch weight was positively correlated with $\mathrm{K}$ content in bloom and 
negatively with $\mathrm{Mg}$ content in most of the samples. In this way, $\mathrm{K}$ to $\mathrm{Ca}, \mathrm{K}$ to $\mathrm{Mg}$ and $\mathrm{Ca}+\mathrm{Mg}$ to $\mathrm{K}$ ratios calculated in petioles at bloom were negatively correlated with bunch weight. $\mathrm{K}$ content and $\mathrm{Ca}$ to $\mathrm{Mg}$ ratio analysed in petioles together with $\mathrm{Fe}$ content in blades at véraison were positively correlated with $\mathrm{pH}$. B content analysed in bloom and $\mathrm{Ca}$ to $\mathrm{Mg}$ ratio calculated in petioles in both phenological stages were correlated with plant yield.

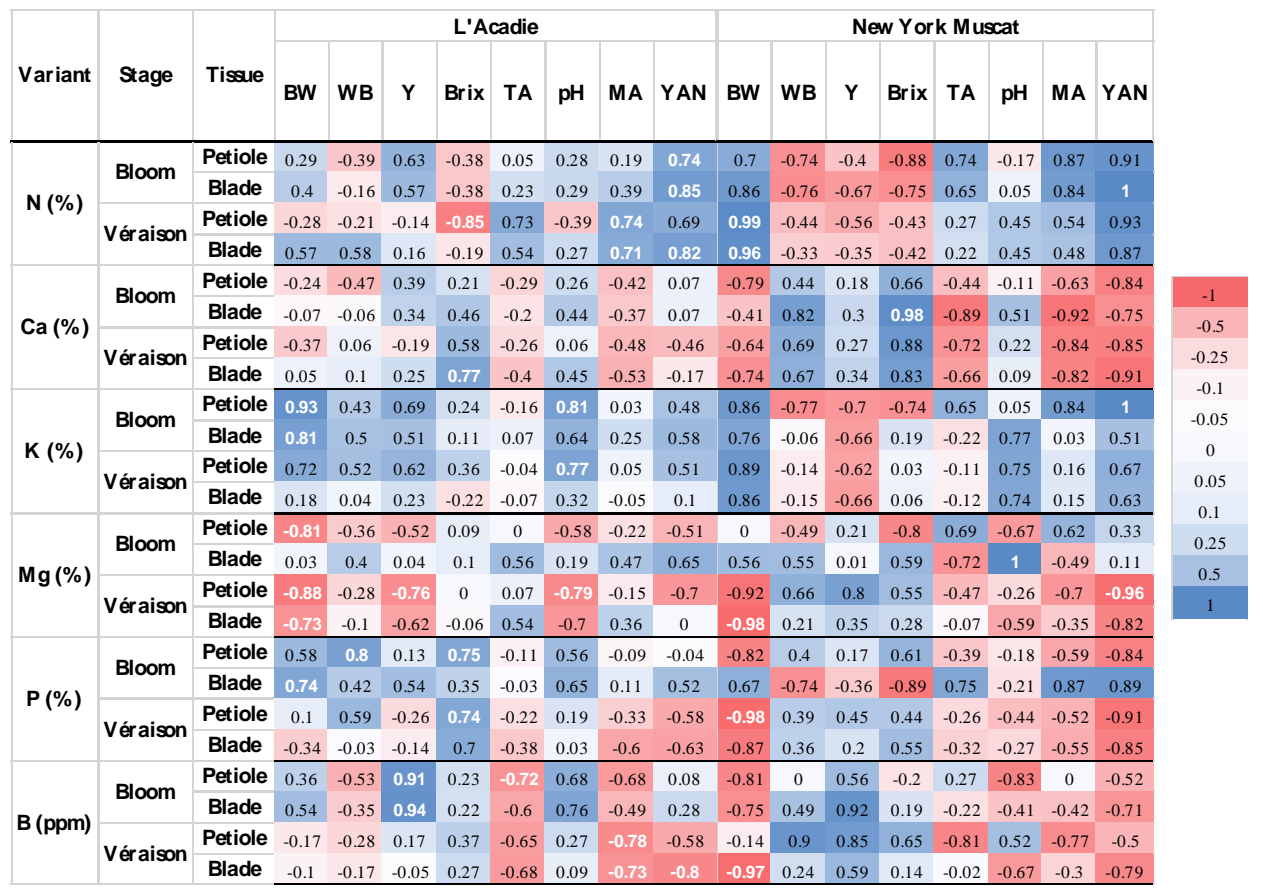

Figure 1. Correlation coefficients between yield, yield components and some must parameters versus nutrient concentrations of blades and petioles analysed at bloom and véraison stages of the interspecific hybrids ("L'Acadie" and "New York Muscat") cultivated in Annapolis Valley ( $\mathrm{n}=9,6$, respectively). The boxes in blue show positive correlations, while the boxes in red show negative correlations $(-1<\mathrm{r}<1)$. BW: bunch weight. WB: weight of berries. Y: Yield per plant. TA: Total acidity.

MA: Malic acid.

\section{Linear correlations in "New York Muscat"}

Linear correlations between nutrient status and yield, yield components and must parameters in "New York Muscat" is shown in Figures 1 and 2. From this, it is possible to observe that the determined correlations were more pronounced in "New York Muscat" than in "L'Acadie". Bunch weight was positively correlated with $\mathrm{N}$ levels determined at véraison and negatively correlated with $\mathrm{K}$ to $\mathrm{Ca}$ ratio calculated in this same phenological stage. In addition, $\mathrm{Mg}$ and $\mathrm{B}$ content together with $\mathrm{Ca}+\mathrm{Mg}$ to $\mathrm{K}$ ratio determined in blade at véraison, as well as $\mathrm{P}$ petiole content measured at this phenological stage were negatively correlated with bunch weight. Conversely, Fe petiole content analysed at bloom was positively correlated with bunch weight and yeast assimilable nitrogen. This last parameter was negatively correlated with $\mathrm{Fe}$ petiole content measured at véraison and with $\mathrm{K}$ to $\mathrm{Ca}$ and $\mathrm{Ca}+\mathrm{Mg}$ to $\mathrm{K}$ ratios in petioles at bloom. $\mathrm{K}$ to $\mathrm{Mg}$ ratio calculated in blades at bloom was positively correlated with weight of berries, while $\mathrm{K}$ to $\mathrm{Ca}$ and $\mathrm{Ca}+\mathrm{Mg}$ to $\mathrm{K}$ ratios had the opposite effect on malic acid. $\mathrm{Zn}$ content analysed in bloom was positively correlated with plant yield.

\section{Linear correlations in "Chardonnay"}

Linear correlations between nutrient status and yield, yield components and must parameters in "Chardonnay" is shown in Figures 3 and 4. N petiole content measured in bloom was negatively correlated with soluble solids and positively with malic acid, whereas $\mathrm{N}$ petiole content measured at véraison was negatively correlated with yield and $\mathrm{pH}$, and positively with yeast assimilable nitrogen. In this way, Fe petiole content determined at véraison was positively correlated with bunch weight and weight of berries but negatively correlated with total acidity and yeast assimilable nitrogen. For its part, total acidity was negatively correlated with $\mathrm{Ca}$ content in blades measured at bloom, as well as with $\mathrm{Zn}$ content determined in bloom and the $\mathrm{K}$ to $\mathrm{Mg}$ ratio calculated in petioles at bloom. Malic acid was negatively correlated with $\mathrm{Zn}$ content and $\mathrm{K}$ to $\mathrm{Mg}$ 
ratio analysed in petioles at bloom. Bunch weight was positively correlated with $\mathrm{Zn}$ content measured at bloom and $\mathrm{Fe}$ petiole content determined in véraison, and with $\mathrm{B}$ content in the blades determined at bloom.

\begin{tabular}{|c|c|c|c|c|c|c|c|c|c|c|c|c|c|c|c|c|c|c|c|}
\hline \multirow[b]{2}{*}{ Variant } & \multirow[b]{2}{*}{ Stage } & \multirow[b]{2}{*}{ Tissue } & \multicolumn{8}{|c|}{ L'Acadie } & \multicolumn{8}{|c|}{ New York Muscat } & \\
\hline & & & BW & WB & $\mathbf{Y}$ & Brix & TA & $\mathrm{pH}$ & MA & YAN & BW & WB & $\mathbf{Y}$ & Brix & TA & $\mathrm{pH}$ & MA & YAN & \\
\hline \multirow{4}{*}{$\mathrm{Fe}(\mathrm{ppm})$} & \multirow{2}{*}{ Bloom } & Petiole & 0.5 & -0.12 & 0.68 & -0.02 & -0.06 & 0.48 & 0.08 & 0.65 & 0.96 & -0.58 & -0.63 & -0.57 & 0.43 & 0.3 & 0.67 & 0.98 & \\
\hline & & Blade & -0.13 & -0.6 & 0.34 & -0.55 & 0.2 & -0.09 & 0.25 & 0.64 & 0.7 & -0.66 & -0.3 & -0.84 & 0.67 & -0.13 & 0.81 & 0.88 & \\
\hline & \multirow{2}{*}{ Véraison } & Petiole & -0.47 & -0.24 & -0.15 & -0.48 & 0.35 & -0.21 & 0.23 & 0.29 & -0.75 & 0.85 & 0.65 & 0.86 & -0.78 & 0.14 & -0.92 & -0.97 & \\
\hline & & Blade & 0.57 & 0.24 & 0.62 & 0.06 & -0.01 & 0.73 & 0.1 & 0.71 & -0.2 & 0.91 & 0.39 & 0.99 & -0.98 & 0.71 & -0.95 & -0.63 & \\
\hline \multirow{4}{*}{$\mathrm{Zn}$ (ppm) } & \multirow{2}{*}{ Bloom } & Petiole & -0.06 & 0.56 & -0.64 & -0.02 & 0.25 & -0.32 & 0.23 & -0.41 & -0.4 & 0.82 & 0.98 & 0.5 & -0.63 & 0.17 & -0.7 & -0.63 & -1 \\
\hline & & Blade & -0.13 & -0.09 & 0.17 & 0.55 & -0.51 & 0.34 & -0.69 & -0.47 & -0.44 & 0.82 & 0.99 & 0.51 & -0.63 & 0.15 & -0.7 & -0.66 & -0.5 \\
\hline & \multirow{2}{*}{ Véraison } & Petiole & 0.69 & 0.77 & 0.03 & -0.05 & 0.21 & 0.36 & 0.43 & 0.35 & -0.34 & 0.93 & 0.92 & 0.68 & -0.8 & 0.37 & -0.83 & -0.66 & -0.25 \\
\hline & & Blade & -0.21 & -0.41 & 0.16 & 0.28 & -0.7 & 0.18 & -0.81 & -0.65 & -0.37 & 0.91 & 0.94 & 0.65 & -0.77 & 0.32 & -0.81 & -0.66 & -0.1 \\
\hline \multirow{4}{*}{$\mathrm{Ca} / \mathrm{Mg}$} & \multirow{2}{*}{ Bloom } & Petiole & 0.63 & \begin{tabular}{l|l}
-0.08 \\
\end{tabular} & 0.93 & 0.12 & -0.3 & 0.86 & -0.2 & 0.58 & -0.36 & 0.43 & -0.15 & 0.76 & -0.57 & 0.33 & -0.62 & -0.56 & -0.05 \\
\hline & & Blade & -0.12 & -0.38 & 0.39 & 0.47 & -0.68 & 0.4 & -0.82 & -0.42 & -0.71 & 0.67 & 0.32 & 0.84 & -0.68 & 0.13 & -0.82 & -0.89 & 0 \\
\hline & \multirow{2}{*}{ Véraison } & Petiole & 0.81 & 0.35 & 0.76 & 0.46 & -0.38 & 0.96 & -0.25 & 0.36 & 0.67 & -0.13 & -0.74 & 0.18 & -0.16 & 0.68 & 0.06 & 0.47 & 0.05 \\
\hline & & Blade & 0.55 & 0.22 & 0.58 & 0.72 & -0.63 & 0.8 & -0.61 & -0.12 & 0.33 & 0.64 & -0.01 & 0.78 & -0.83 & 0.96 & -0.65 & -0.13 & 0.1 \\
\hline \multirow{4}{*}{$\mathrm{K} / \mathrm{Ca}$} & \multirow{2}{*}{ Bloom } & Petiole & -0.71 & -0.44 & -0.28 & 0.24 & -0.23 & -0.33 & -0.45 & -0.51 & -0.83 & 0.71 & 0.51 & 0.79 & -0.65 & -0.01 & -0.83 & -0.97 & 0.125 \\
\hline & & Blade & -0.44 & -0.28 & 0.01 & 0.4 & -0.27 & 0.05 & -0.49 & -0.32 & -0.61 & 0.86 & 0.5 & 0.95 & -0.86 & 0.33 & -0.96 & -0.9 & 0.5 \\
\hline & \multirow{2}{*}{ Véraison } & Petiole & -0.58 & -0.41 & -0.27 & 0.21 & -0.31 & -0.27 & -0.48 & -0.68 & -0.97 & 0.36 & 0.68 & 0.23 & -0.13 & -0.58 & -0.4 & -0.84 & 1 \\
\hline & & Blade & -0.03 & 0 & 0.15 & 0.65 & -0.3 & 0.23 & -0.39 & -0.11 & -0.97 & 0.46 & 0.71 & 0.36 & -0.25 & -0.48 & -0.52 & -0.9 & \\
\hline \multirow{4}{*}{$\mathrm{K} / \mathrm{Mg}$} & \multirow{2}{*}{ Bloom } & Petiole & -0.78 & -0.38 & -0.48 & 0.19 & -0.16 & -0.5 & -0.38 & -0.64 & -0.6 & 0.13 & 0.76 & -0.2 & 0.15 & -0.63 & -0.04 & -0.41 & \\
\hline & & Blade & -0.62 & -0.11 & -0.33 & 0.16 & 0.24 & -0.29 & 0.01 & -0.06 & -0.14 & 0.96 & 0.75 & 0.8 & -0.92 & 0.64 & -0.88 & -0.55 & \\
\hline & \multirow{2}{*}{ Véraison } & Petiole & -0.66 & -0.54 & -0.46 & -0.14 & -0.13 & -0.62 & -0.25 & -0.67 & -0.85 & 0.24 & 0.74 & 0.02 & 0.03 & -0.67 & -0.23 & -0.67 & \\
\hline & & Blade & -0.54 & -0.2 & -0.43 & -0.02 & 0.34 & -0.61 & 0.24 & -0.01 & -0.89 & 0.06 & 0.54 & -0.09 & 0.19 & -0.81 & -0.09 & -0.63 & \\
\hline \multirow{4}{*}{$\begin{array}{c}(\mathrm{Ca}+\mathrm{Mg}) \\
\mathrm{K}\end{array}$} & \multirow{2}{*}{ Bloom } & Petiole & -0.74 & -0.42 & -0.35 & 0.23 & -0.2 & -0.39 & -0.43 & -0.55 & -0.9 & 0.7 & 0.64 & 0.71 & -0.58 & -0.12 & -0.79 & -1 & \\
\hline & & Blade & -0.47 & -0.26 & -0.03 & 0.38 & -0.22 & 0.01 & -0.44 & -0.3 & -0.59 & 0.88 & 0.52 & 0.96 & -0.87 & 0.35 & -0.97 & -0.89 & \\
\hline & \multirow{2}{*}{ Véraison } & Petiole & -0.64 & -0.49 & -0.37 & 0.05 & -0.24 & -0.47 & -0.39 & -0.71 & -0.94 & 0.32 & 0.7 & 0.16 & -0.08 & -0.62 & -0.35 & -0.79 & \\
\hline & & Blade & -0.11 & -0.03 & 0.08 & 0.6 & -0.22 & 0.12 & -0.32 & -0.1 & -0.97 & 0.4 & 0.69 & 0.29 & -0.18 & -0.54 & -0.45 & -0.87 & \\
\hline
\end{tabular}

Figure 2. Correlation coefficients between yield, yield components and some must parameters versus nutrient concentrations and their ratios of blades and petioles analysed at bloom and véraison stages of the interspecific hybrids ("L'Acadie" and

"New York Muscat") cultivated in Annapolis Valley ( $n=9,6$, respectively). The blank coefficients show significant differences from 0.05 level. The boxes in blue show positive correlations, while the boxes in red show negative correlations

$(-1<\mathrm{r}<1)$. BW: bunch weight. WB: weight of berries. Y: Yield per plant. TA: Total acidity. MA: Malic acid.

\begin{tabular}{|c|c|c|c|c|c|c|c|c|c|c|c|c|c|c|c|c|c|c|c|c|c|c|c|c|c|c|c|}
\hline \multirow{2}{*}{ Variant } & \multirow[b]{2}{*}{ Stage } & \multirow[b]{2}{*}{ Tissue } & \multicolumn{8}{|c|}{ Chardonnay } & \multicolumn{8}{|c|}{ Riesing } & \multicolumn{8}{|c|}{ Pinot Noir } & \\
\hline & & & BW & WB & $\mathbf{Y}$ & Brix & TA & pH & MA & YAN & BW & WB & $\mathbf{Y}$ & Brix & TA & $\mathrm{pH}$ & MA & YAN & BW & WB & $\mathbf{Y}$ & Brix & TA & pH & MA & YAN & \\
\hline \multirow{4}{*}{$\mathbf{N}(\%)$} & \multirow{2}{*}{ Bloom } & Petiole & -0.55 & -0.82 & -0.75 & -0.92 & 0.83 & -0.44 & 0.94 & 0.76 & -0.65 & -0.16 & -0.79 & -0.1 & 0.71 & -0.3 & 0.76 & 0.72 & 0.98 & -0.21 & -0.2 & -0.86 & 0.8 & 0.22 & 0.99 & 0.94 & \\
\hline & & Blade & 0.36 & 0.05 & -0.27 & 0.29 & -0.29 & -0.34 & -0.33 & 0.13 & -0.67 & -0.69 & -0.69 & -0.27 & 0.81 & -0.43 & 0.76 & 0.61 & 0.96 & -0.45 & 0.13 & -0.65 & 0.87 & -0.08 & 0.95 & 0.82 & \\
\hline & \multirow{2}{*}{ Véraison } & Petiole & -0.53 & -0.79 & -0.89 & -0.54 & 0.69 & -0.88 & 0.61 & 0.92 & -0.51 & -0.25 & -0.54 & -0.59 & 0.44 & 0.42 & 0.52 & 0.74 & 0.95 & -0.26 & -0.33 & -0.92 & 0.54 & 0.47 & 0.89 & 1 & \\
\hline & & Blade & -0.7 & -0.42 & -0.39 & -0.09 & 0.66 & -0.54 & 0.48 & 0.69 & 0.58 & 0.46 & 0.69 & 0.91 & -0.68 & -0.1 & -0.71 & -0.73 & 0.67 & 0.35 & -0.41 & -0.73 & 0.82 & 0.19 & 0.84 & 0.67 & \\
\hline \multirow{4}{*}{$\mathrm{Ca}(\%)$} & \multirow{2}{*}{ Bloom } & Petiole & 0.77 & 0.49 & 0.41 & 0.31 & -0.85 & 0.35 & -0.78 & -0.75 & -0.09 & 0.24 & 0.13 & -0.04 & -0.31 & 0.61 & -0.21 & 0.13 & -0.23 & -0.43 & 0.04 & 0.17 & -0.77 & 0.32 & -0.48 & -0.11 & \\
\hline & & Blade & 0.83 & 0.6 & 0.5 & 0.42 & -0.91 & 0.45 & -0.83 & -0.81 & 0.02 & 0.6 & 0.3 & 0 & -0.54 & 0.1 & -0.46 & -0.39 & -0.48 & -0.51 & 0.41 & 0.58 & -0.76 & -0.11 & -0.7 & -0.48 & -1 \\
\hline & \multirow{2}{*}{ Véraison } & Petiole & 0.24 & 0.33 & 0.17 & 0.63 & -0.43 & -0.16 & -0.6 & -0.17 & 0.59 & 0.8 & 0.67 & 0.89 & -0.78 & -0.12 & -0.76 & -0.78 & 0.2 & -0.81 & 0.32 & 0.01 & -0.34 & 0.09 & -0.08 & 0.18 & -0.5 \\
\hline & & Blade & -0.16 & 0.12 & 0.26 & -0.11 & 0.27 & 0.55 & 0.41 & -0.04 & -0.17 & 0.64 & -0.12 & 0.5 & -0.1 & -0.54 & -0.03 & -0.17 & -0.44 & -0.61 & 0.91 & 0.86 & -0.24 & -0.79 & -0.54 & -0.66 & -0.25 \\
\hline \multirow{4}{*}{ K (\%) } & \multirow{2}{*}{ Bloom } & Petiole & -0.24 & -0.2 & -0.38 & 0.13 & 0.33 & -0.41 & 0.24 & 0.58 & -0.5 & 0.18 & -0.62 & -0.58 & 0.45 & -0.26 & 0.52 & 0.41 & 0.77 & 0.15 & -0.7 & -1 & 0.36 & 0.75 & 0.76 & 0.93 & -0.1 \\
\hline & & Blade & -0.58 & -0.19 & 0.1 & -0.34 & 0.55 & 0.2 & 0.57 & 0.15 & -0.1 & 0.44 & -0.22 & -0.1 & 0.03 & 0.15 & 0.13 & 0.22 & 0.41 & 0.27 & -0.86 & -0.82 & -0.15 & 0.97 & 0.34 & 0.68 & -0.05 \\
\hline & \multirow{2}{*}{ Véraison } & Petiole & -0.06 & -0.21 & -0.13 & -0.53 & 0.32 & 0.29 & 0.54 & 0.11 & 0.06 & 0.67 & 0.23 & 0.06 & -0.48 & 0.21 & -0.38 & -0.25 & 0.37 & 0.18 & -0.8 & -0.76 & -0.25 & 0.96 & 0.27 & 0.64 & 0 \\
\hline & & Blade & -0.21 & -0.59 & -0.54 & -0.84 & 0.53 & -0.15 & 0.72 & 0.44 & 0.09 & 0.55 & -0.12 & -0.18 & -0.04 & -0.07 & 0.03 & -0.03 & 0.33 & 0.48 & -0.95 & -0.81 & -0.12 & 0.97 & 0.33 & 0.62 & 0.05 \\
\hline \multirow{4}{*}{$\mathbf{M g}(\%)$} & \multirow{2}{*}{ Bloom } & Petiole & 0.63 & 0.53 & 0.29 & 0.69 & -0.73 & 0.09 & -0.8 & -0.42 & -0.09 & -0.41 & 0.03 & 0.03 & 0.12 & -0.36 & 0.03 & -0.14 & -0.74 & -0.19 & 0.73 & 0.99 & -0.32 & -0.78 & -0.73 & -0.92 & 0.1 \\
\hline & & Blade & -0.53 & -0.02 & 0.31 & -0.12 & 0.39 & 0.3 & 0.36 & -0.04 & 0.03 & -0.21 & 0.23 & 0.19 & -0.13 & -0.3 & -0.21 & -0.34 & -0.8 & -0.12 & 0.67 & 1 & -0.41 & -0.71 & -0.8 & -0.95 & 0.25 \\
\hline & \multirow{2}{*}{ Véraison } & Petiole & -0.31 & -0.2 & -0.3 & 0.19 & 0.2 & -0.58 & -0.01 & 0.42 & -0.4 & -0.1 & -0.17 & 0.1 & 0.14 & -0.54 & 0.11 & -0.07 & -0.67 & -0.27 & 0.9 & 0.97 & -0.25 & -0.83 & -0.67 & -0.87 & 0.5 \\
\hline & & Blade & -0.66 & -0.45 & -0.45 & -0.16 & 0.72 & -0.47 & 0.61 & 0.76 & -0.87 & -0.19 & -0.73 & -0.38 & 0.6 & -0.59 & 0.63 & 0.45 & -0.63 & 0.11 & 0.6 & 0.84 & 0.01 & -0.82 & -0.5 & -0.83 & 1 \\
\hline \multirow{4}{*}{$\mathbf{P}(\%)$} & \multirow{2}{*}{ Bloom } & Petiole & -0.05 & -0.02 & 0.24 & -0.23 & -0.18 & 0.06 & -0.21 & -0.43 & -0.41 & 0.1 & -0.51 & -0.2 & 0.36 & 0.14 & 0.45 & 0.59 & 0.21 & -0.37 & 0.75 & 0.3 & 0.72 & -0.89 & 0.3 & -0.11 & \\
\hline & & Blade & -0.31 & 0.42 & 0.67 & 0.46 & 0.02 & 0.47 & -0.11 & -0.33 & -0.58 & 0.21 & -0.72 & -0.42 & 0.56 & -0.68 & 0.6 & 0.33 & 0.4 & -0.44 & 0.7 & 0.12 & 0.82 & -0.8 & 0.47 & 0.09 & \\
\hline & \multirow{2}{*}{ Véraison } & Petiole & -0.06 & 0.54 & 0.79 & 0.41 & -0.17 & 0.7 & -0.19 & -0.55 & -0.69 & 0.11 & -0.55 & -0.16 & 0.33 & -0.12 & 0.42 & 0.5 & -0.57 & 0.26 & 0.47 & 0.72 & 0.12 & -0.76 & -0.38 & -0.74 & \\
\hline & & Blade & -0.11 & 0.58 & 0.83 & 0.56 & -0.23 & 0.59 & -0.34 & -0.57 & -0.42 & 0.44 & -0.42 & -0.38 & 0.17 & -0.29 & 0.26 & 0.16 & -0.61 & 0.08 & 0.62 & 0.84 & 0.03 & -0.84 & -0.48 & -0.81 & \\
\hline \multirow{4}{*}{ B (ppm) } & Bloom & Petiole & $\begin{array}{l}-0.06 \\
\end{array}$ & -0.46 & -0.72 & -0.35 & 0.45 & -0.39 & 0.57 & 0.74 & -0.67 & -0.16 & -0.67 & -0.13 & 0.62 & -0.82 & 0.61 & 0.31 & 0.8 & -0.38 & 0.32 & -0.41 & 0.97 & -0.38 & 0.86 & 0.59 & \\
\hline & Bloom & Blade & 0.97 & 0.74 & 0.43 & 0.64 & -0.83 & 0.62 & -0.67 & -0.59 & 0.33 & 0.04 & 0.66 & 0.11 & -0.63 & 0.24 & -0.67 & -0.6 & -0.3 & -0.75 & 0.9 & 0.74 & -0.24 & -0.7 & -0.45 & -0.52 & \\
\hline & & Petiole & -0.41 & -0.58 & -0.52 & -0.75 & 0.72 & -0.12 & 0.88 & 0.59 & -0.69 & -0.72 & -0.49 & -0.79 & 0.49 & 0.51 & 0.52 & 0.79 & 0 & -0.92 & 0.8 & 0.45 & -0.17 & -0.49 & -0.22 & -0.19 & \\
\hline & & Blade & -0.1 & 0.17 & 0.38 & -0.12 & 0.13 & 0.59 & 0.25 & -0.24 & -0.35 & -0.51 & -0.52 & -0.63 & 0.65 & -0.41 & 0.59 & 0.34 & -0.59 & -0.45 & 0.85 & 0.94 & -0.31 & -0.79 & -0.66 & -0.79 & \\
\hline
\end{tabular}

Figure 3. Correlation coefficients between yield, yield components and some must parameters versus nutrient concentrations of blades and petioles analysed at bloom and véraison stages of the Vitis vinifera varieties ("Chardonnay", "Riesling" and "Pinot Noir") cultivated in Annapolis Valley ( $\mathrm{n}=6,9,4$, respectively). The boxes in blue show positive correlations, while the boxes in red show negative correlations $(-1<r<1)$. BW: bunch weight. WB: weight of berries. Y: Yield per plant. TA: Total acidity. MA: Malic acid. 


\section{Linear correlations in "Riesling"}

Linear correlations between nutrient status and yield, yield components and must parameters in "Riesling" is shown in Figures 3 and 4. From this, it is possible to observe that the determined relationships were less pronounced in "Riesling" than in the rest of the Vitis vinifera varieties. Furthermore, there were few linear correlations between nutrient status and yield, yield components and must parameters in
"Riesling". In this way, soluble solids were positively correlated with $\mathrm{N}$ content in blades determined at véraison and $\mathrm{Ca}$ content in petioles measured at this same phenological stage. $\mathrm{pH}$ was negatively correlated with $\mathrm{B}$ petiole content determined at bloom. Bunch weight was positively correlated with $\mathrm{Ca}$ to $\mathrm{Mg}$ ratio calculated in blades at véraison and negatively with $\mathrm{Mg}$ blade content measured at this same phenological stage.

\begin{tabular}{|c|c|c|c|c|c|c|c|c|c|c|c|c|c|c|c|c|c|c|c|c|c|c|c|c|c|c|c|}
\hline \multirow{2}{*}{ Variant } & \multirow{2}{*}{ Stage } & \multirow{2}{*}{ Tissue } & \multicolumn{8}{|c|}{ Chardonnay } & \multicolumn{8}{|c|}{ Riesing } & \multicolumn{8}{|c|}{ Pinot Noir } & \\
\hline & & & BW & WB & $Y$ & Brix & TA & $\mathrm{pH}$ & MA & YAN & BW & WB & $Y$ & Brix & TA & $\mathrm{pH}$ & MA & YAN & BW & WB & $Y$ & Brix & TA & $\mathrm{pH}$ & MA & YAN & \\
\hline \multirow{4}{*}{$\mathrm{Fe}(\mathrm{ppm})$} & \multirow{2}{*}{ Bloom } & Petiole & 56 & 0.38 & 0.07 & 59 & .53 & -0.01 & -0.56 & -0.15 & 0.4 & -0.09 & 0.68 & 0.23 & -0.57 & 0.23 & -0.64 & -0.57 & 0.65 & -0.03 & -0.65 & -0.89 & 0.05 & 0.84 & 0.55 & 85 & \\
\hline & & Blade & 0.76 & 0.25 & -0.08 & 0.27 & -0.62 & -0.02 & -0.53 & -0.23 & 0.45 & 0.01 & 0.71 & 0.3 & -0.62 & 0.14 & -0.68 & -0.66 & 0.09 & -0.11 & -0.49 & -0.38 & -0.59 & 0.79 & -0.11 & 0.33 & \\
\hline & \multirow{2}{*}{ Véraison } & Petiole & 0.91 & 0.91 & 0.78 & 0.77 & -0.98 & 0.77 & -0.9 & -0.92 & 0.48 & -0.01 & 0.66 & 0.4 & -0.54 & 0.01 & -0.62 & -0.65 & -0.68 & -0.24 & 0.24 & 0.64 & -0.9 & -0.01 & -0.86 & -0.62 & \\
\hline & & Blade & 0.46 & 0.29 & 0.38 & -0.07 & -0.45 & 0.52 & -0.29 & -0.62 & 0.4 & -0.02 & 0.48 & 0.52 & -0.34 & -0.26 & -0.43 & -0.57 & -0.39 & 0.08 & -0.41 & 0.03 & -0.9 & 0.64 & -0.55 & -0.14 & \\
\hline \multirow{4}{*}{ Zn (ppm) } & \multirow{2}{*}{ Bloom } & Petiole & 0.92 & 0.72 & 0.56 & 0.58 & -0.97 & 0.54 & -0.89 & 0.83 & -0.07 & 0.34 & -0.19 & -0.45 & 0.04 & 0.08 & 0.11 & 0.11 & -0.19 & -0.05 & -0.41 & -0.12 & -0.79 & 0.7 & -0.38 & 0.06 & \\
\hline & & Blade & 0.99 & 0.71 & 0.42 & 0.59 & -0.88 & 0.56 & -0.73 & -0.65 & 0.27 & 0.54 & 0.06 & -0.07 & -0.19 & 0.14 & -0.12 & -0.1 & 0.98 & -0.52 & -0.03 & -0.77 & 0.62 & 0.21 & 0.88 & 0.93 & -1 \\
\hline & \multirow{2}{*}{ Véraison } & Petiole & 0.49 & 0.04 & -0.08 & 0.01 & -0.54 & -0.23 & -0.55 & -0.31 & 0.67 & 0.75 & 0.57 & 0.3 & -0.68 & 0.25 & -0.64 & -0.58 & 0.04 & -0.83 & 0.46 & 0.22 & -0.4 & -0.07 & -0.23 & -0.01 & -0.5 \\
\hline & & Blade & -0.21 & -0.55 & -0.78 & -0.33 & 0.5 & -0.61 & 0.52 & 0.82 & 0.61 & 0.55 & 0.33 & 0.31 & -0.36 & 0.22 & -0.33 & -0.28 & 0.89 & -0.76 & 0.32 & -0.5 & 0.64 & -0.12 & 0.77 & 0.74 & -0.25 \\
\hline \multirow{4}{*}{$\mathrm{Ca} / \mathrm{Mg}$} & \multirow{2}{*}{ Bloom } & Petiole & -0.35 & -0.47 & -0.27 & -0.74 & 0.48 & -0.02 & 0.61 & 0.22 & 0.23 & 0.54 & 0.11 & 0.14 & -0.26 & 0.33 & -0.18 & -0.02 & 0.66 & -0.07 & -0.63 & -0.88 & 0.04 & 0.83 & 0.54 & 0.85 & -0.1 \\
\hline & & Blade & 0.83 & 0.41 & 0.14 & 0.38 & -0.79 & 0.13 & -0.72 & -0.47 & 0.11 & 0.49 & -0.03 & -0.06 & -0.14 & 0.25 & -0.05 & 0.07 & 0.15 & -0.46 & -0.14 & -0.22 & -0.53 & 0.52 & -0.11 & 0.29 & -0.05 \\
\hline & \multirow{2}{*}{ Véraison } & Petiole & 0.4 & 0.24 & 0.29 & -0.15 & -0.25 & 0.61 & -0.02 & -0.43 & 0.69 & 0.57 & 0.49 & 0.41 & -0.52 & 0.37 & -0.48 & -0.37 & 0.79 & -0.08 & -0.58 & -0.95 & 0.26 & 0.74 & 0.71 & 0.94 & 0 \\
\hline & & Blade & 0.7 & 0.51 & 0.5 & 0.18 & -0.7 & 0.61 & -0.55 & -0.77 & 0.86 & 0.59 & 0.7 & 0.62 & -0.7 & 0.37 & -0.69 & -0.57 & 0.6 & -0.35 & -0.39 & -0.7 & -0.09 & 0.69 & 0.41 & 0.75 & 0.05 \\
\hline \multirow{4}{*}{$\mathrm{K} / \mathrm{Ca}$} & \multirow{2}{*}{ Bloom } & Petiole & 0.44 & 0.24 & 0.33 & -0.06 & -0.52 & 0.32 & -0.43 & -0.63 & 0.44 & -0.2 & 0.66 & 0.48 & -0.52 & 0.44 & -0.57 & -0.37 & -0.47 & -0.6 & 0.87 & 0.86 & -0.29 & -0.75 & -0.58 & -0.68 & 0.1 \\
\hline & & Blade & 0.77 & 0.4 & 0.17 & 0.4 & -0.79 & 0.08 & -0.77 & -0.5 & 0.22 & -0.1 & 0.47 & 0.18 & -0.38 & -0.1 & -0.45 & -0.52 & -0.51 & -0.53 & 0.89 & 0.91 & -0.25 & -0.81 & -0.59 & -0.73 & 0.25 \\
\hline & \multirow{2}{*}{ Véraison } & Petiole & -0.1 & -0.07 & -0.15 & 0.26 & -0.12 & -0.55 & -0.34 & 0.12 & 0.11 & -0.3 & -0.03 & 0.37 & 0.23 & -0.45 & 0.13 & -0.08 & -0.47 & -0.39 & 0.9 & 0.89 & -0.01 & -0.94 & -0.46 & -0.73 & 0.5 \\
\hline & & Blade & 0.1 & 0.74 & 0.82 & 0.84 & -0.36 & 0.61 & -0.47 & -0.52 & -0.14 & -0.2 & 0.05 & 0.45 & 0 & -0.21 & -0.03 & -0.04 & -0.38 & -0.6 & 0.95 & 0.84 & -0.1 & -0.87 & -0.46 & -0.64 & 1 \\
\hline \multirow{4}{*}{$\mathrm{K} / \mathrm{Mg}$} & \multirow{2}{*}{ Bloom } & Petiole & 0.73 & 0.6 & 0.54 & 0.5 & -0.91 & 0.36 & -0.9 & -0.83 & 0.26 & -0.29 & 0.42 & 0.35 & -0.25 & -0.04 & -0.34 & -0.37 & -0.49 & -0.55 & 0.9 & 0.89 & -0.24 & -0.81 & -0.57 & -0.71 & \\
\hline & & Blade & 0.58 & 0.36 & 0.16 & 0.47 & -0.71 & -0.08 & -0.78 & -0.42 & 0.15 & -0.23 & 0.3 & 0.22 & -0.16 & -0.25 & -0.25 & -0.39 & -0.57 & -0.27 & 0.84 & 0.93 & -0.08 & -0.91 & -0.54 & -0.81 & \\
\hline & \multirow{2}{*}{ Véraison } & Petiole & -0.21 & -0.18 & -0.27 & 0.19 & 0.04 & -0.62 & -0.18 & 0.29 & 0.69 & 0.57 & 0.49 & 0.41 & -0.52 & 0.37 & -0.48 & -0.37 & -0.55 & -0.3 & 0.85 & 0.92 & -0.08 & -0.91 & -0.53 & -0.8 & \\
\hline & & Blade & -0.54 & -0.21 & -0.22 & 0.13 & 0.49 & -0.37 & 0.32 & 0.55 & -0.69 & -0.4 & -0.51 & -0.13 & 0.49 & -0.46 & 0.48 & 0.37 & -0.56 & -0.2 & 0.81 & 0.91 & -0.03 & -0.92 & -0.51 & -0.81 & \\
\hline \multirow{4}{*}{$\begin{array}{c}(\mathrm{Ca}+\mathrm{Mg}) / \\
\mathrm{K}\end{array}$} & Bloom & Petiole & 0.53 & 0.34 & 0.4 & 0.08 & -0.64 & 0.35 & -0.58 & -0.72 & 0.39 & -0.25 & 0.6 & 0.44 & -0.44 & 0.28 & -0.5 & -0.38 & -0.47 & -0.59 & 0.89 & 0.87 & -0.27 & -0.77 & -0.57 & -0.69 & \\
\hline & Bloom & Blade & 0.76 & 0.4 & 0.17 & 0.41 & 0.79 & 0.06 & -0.77 & -0.49 & 0.19 & -0.15 & 0.41 & 0.2 & -0.3 & -0.16 & -0.38 & -0.48 & -0.53 & -0.49 & 0.89 & 0.92 & -0.23 & -0.83 & -0.59 & -0.75 & \\
\hline & & Petiole & -0.15 & -0.12 & -0.2 & 0.23 & -0.05 & -0.59 & -0.27 & 0.19 & -0.02 & -0.36 & -0.12 & 0.27 & 0.3 & -0.49 & 0.21 & -0.01 & -0.51 & -0.35 & 0.88 & 0.91 & -0.05 & -0.93 & -0.5 & -0.76 & \\
\hline & & Blade & -0.13 & 0.49 & 0.54 & 0.71 & -0.08 & 0.32 & -0.24 & -0.18 & -0.27 & -0.26 & -0.08 & 0.33 & 0.12 & -0.27 & 0.09 & 0.05 & -0.42 & -0.55 & 0.94 & 0.87 & $\begin{array}{r}-0.09 \\
\end{array}$ & -0.89 & -0.47 & -0.68 & \\
\hline
\end{tabular}

Figure 4. Correlation coefficients between yield, yield components and some must parameters versus nutrient concentrations and their ratios of blades and petioles analysed at bloom and véraison stages of the Vitis vinifera varieties ("Chardonnay",

"Riesling" and "Pinot Noir") cultivated in Annapolis Valley ( $\mathrm{n}=6,9,4$, respectively). The boxes in blue show positive correlations, while the boxes in red show negative correlations $(-1<\mathrm{r}<1)$. BW: bunch weight. WB: weight of berries. Y: Yield per plant. TA: Total acidity. MA: Malic acid.

\section{Linear correlations in "Pinot Noir"}

Linear correlations between nutrient status and yield, yield components and must parameters in "Pinot Noir" is shown in Figures 3 and 4. Bunch weight was positively correlated with $\mathrm{N}$ levels in the plant and with $\mathrm{Zn}$ content in blades at bloom. Soluble solids were positively correlated with $\mathrm{Mg}$ levels in the plant and negatively with $\mathrm{K}$ petiole content determined at bloom and $\mathrm{Ca}$ to $\mathrm{Mg}$ ratio calculated in petioles at véraison. $\mathrm{pH}$ was positively correlated with $\mathrm{K}$ levels in the plant, while malic acid was positively correlated with $\mathrm{N}$ levels at bloom. For its part, total acidity was positively correlated with $\mathrm{B}$ petiole content measured at bloom, while yeast assimilable nitrogen content was positively correlated with $\mathrm{N}$ petiole content determined at véraison. $\mathrm{K}$ to $\mathrm{Ca}$ ratio calculated in blades at véraison was positively correlated with yield per plant.

\section{Petiole nutrient content}

As shown in Table 3, the nutrient content measured in bloom varied between 0.7 and $1.6 \%$ for $\mathrm{N}$ ("New
York Muscat" and "Chardonnay", respectively), 1.2 and $1.4 \%$ for Ca ("New York Muscat" and "Pinot Noir", respectively), 1.3 and $2.6 \%$ for $\mathrm{K}$ ("Chardonnay" and "Riesling", respectively), 0.29 and $0.62 \%$ for $\mathrm{Mg}$ ("New York Muscat" and "L'Acadie", respectively), 0.19 and $0.37 \%$ for P, 0.03 and $0.04 \%$ for $\mathrm{Na}, 27$ and $42 \mathrm{mg} / \mathrm{kg}$ for $\mathrm{B}$ ("New York Muscat" and "Riesling", respectively), 31 and $76 \mathrm{mg} / \mathrm{kg}$ for Fe ("New York Muscat" and "Pinot Noir", respectively), and 32 and $59 \mathrm{mg} / \mathrm{kg}$ for Zn ("L'Acadie" and "Chardonnay", respectively). The nutrient content measured in véraison varied between 0.7 and $0.8 \%$ for N ("Riesling" and "Pinot Noir", respectively), 1.5 and $1.8 \%$ for $\mathrm{Ca}$ ("Chardonnay" and "L'Acadie", respectively), 0.6 and $2.6 \%$ for K ("L'Acadie" and "Riesling", respectively), 0.47 and $1.05 \%$ for $\mathrm{Mg}$ ("New York Muscat" and "L'Acadie", respectively), 0.24 and $0.51 \%$ for P ("Pinot Noir" and "Riesling"), 32 and $43 \mathrm{mg} / \mathrm{kg}$ for B ("L'Acadie" and "Riesling", respectively), 26 and $55 \mathrm{mg} / \mathrm{kg}$ for Fe ("New York Muscat" and "Pinot Noir", respectively), and 53 and 
$87 \mathrm{mg} / \mathrm{kg}$ for Zn ("L'Acadie" and "Chardonnay", respectively). Bloom presented higher levels of $\mathrm{N}$ and $\mathrm{Fe}$ in most of the samples compared to véraison which reached higher levels of $\mathrm{Ca}, \mathrm{K}, \mathrm{Mg}, \mathrm{P}, \mathrm{B}$ and Zn (Table 3).

$\mathrm{K}, \mathrm{Mg}$ and $\mathrm{B}$ content in petioles measured in bloom and véraison were significantly affected by variety (Table 3). "Riesling" presented the highest K and B petiole content at bloom and the highest B levels at véraison. "L'Acadie" presented the lowest K petiole levels at véraison and lower B petiole content than the most of the Vitis vinifera varieties at both phenological stages. Generally, "L'Acadie" tended to accumulate higher $\mathrm{Mg}$ petiole levels than the rest of the varieties. $\mathrm{N}$ and $\mathrm{P}$ contents in petioles determined at bloom were also affected by variety, including $\mathrm{Zn}$ petiole content analysed at véraison. "New York Muscat" tended to accumulate low levels of $\mathrm{N}$ and $\mathrm{P}$ in petioles measured at bloom compared to other varieties, while "Chardonnay" showed considerably higher $\mathrm{Zn}$ petiole content than "L'Acadie" and "Riesling".

Season factor significantly affected $\mathrm{N}$ and Ca petiole content determined at bloom and véraison (Tables 2 and 3). Grapevines contained the highest $\mathrm{N}$ petiole content in 2019 season, while in 2017 they presented the highest $\mathrm{Ca}$ petiole content. Season also affected $\mathrm{Zn}$ petiole content analysed at véraison. In 2017 , the grapevines presented the highest $\mathrm{Zn}$ petiole content.
Season and variety interaction affected $\mathrm{Ca}$ and $\mathrm{B}$ petiole content determined at véraison (Tables 2 and 3). B petiole content in "L'Acadie" in 2017 presented higher B content than "New York Muscat", "Chardonnay" and "Pinot Noir" but this variety showed the lowest B content in the following years of study (data not shown). An inverse behaviour was found for the "Chardonnay" variety, which in 2019 presented higher B petiole content than "New York Muscat", "Pinot Noir" and "L'Acadie". Ca petiole content in interspecific hybrids was high in 2017 but towards 2019 they presented lower $\mathrm{Ca}$ petiole content than the $V$. vinifera varieties (data not shown).

\section{Blade nutrient content}

As shown in Table 4, the nutrient content at bloom varied between 2.6 and $3.6 \%$ for $\mathrm{N}$ ("New York Muscat" and "Pinot Noir" , respectively), 1.1 and $1.6 \%$ for $\mathrm{Ca}$ ("Riesling" and "L'Acadie", respectively), 0.9 and $1.5 \%$ for $\mathrm{K}$ ("New York Muscat" and "Chardonnay", respectively), 0.23 and $0.32 \%$ for $\mathrm{Mg}$ ("New York Muscat" and "L'Acadie", respectively), 0.20 and $0.34 \%$ for $\mathrm{P}$ ("New York Muscat" and "Chardonnay", respectively), 23 and $44 \mathrm{mg} / \mathrm{kg}$ for B ("New York Muscat" and "Pinot Noir", respectively), 115 and $261 \mathrm{mg} / \mathrm{kg}$ for Fe ("New York Muscat" and "Riesling", respectively), and 28 and $89 \mathrm{mg} / \mathrm{kg}$ for Zn ("L'Acadie" and "New York Muscat", respectively).

Table IV Nutrient petiole content in bloom and véraison of interspecific hybrids and Vitis vinifera varieties cultivated in Annapolis Valley

\begin{tabular}{|c|c|c|c|c|c|c|c|c|c|c|c|c|c|c|c|c|}
\hline \multirow{2}{*}{ Variety $(V)$} & \multicolumn{2}{|c|}{$\mathbf{N}(\%)$} & \multicolumn{2}{|c|}{$\mathrm{Ca}(\%)$} & \multicolumn{2}{|c|}{$\mathbf{K}(\%)$} & \multicolumn{2}{|c|}{$\operatorname{Mg}(\%)$} & \multicolumn{2}{|c|}{$\mathbf{P}(\%)$} & \multicolumn{2}{|c|}{ B (mg/kg) } & \multicolumn{2}{|c|}{$\mathrm{Fe}(\mathrm{mg} / \mathrm{kg})$} & \multicolumn{2}{|c|}{$\mathrm{Zn}(\mathrm{mg} / \mathrm{kg})$} \\
\hline & Bloom & Véraison & Bloom & Véraison & Bloom & Véraison & Bloom & Véraison & Bloom & Véraison & Bloom & Véraison & Bloom & Véraison & Bloom & Véraison \\
\hline "L'Acadie" & $1.08 \mathrm{ab}$ & $0.74 \mathrm{a}$ & $1.34 \mathrm{ab}$ & $1.77 \mathrm{~b}$ & $1.34 \mathrm{a}$ & $0.61 \mathrm{a}$ & $0.62 \mathrm{c}$ & $1.05 \mathrm{~b}$ & $0.37 \mathrm{~b}$ & $0.41 \mathrm{ab}$ & $30.30 \mathrm{a}$ & $32.39 \mathrm{a}$ & $46.18 \mathrm{ab}$ & $43.22 \mathrm{a}$ & $32.09 \mathrm{a}$ & $52.99 \mathrm{a}$ \\
\hline "NY Muscat" & $0.71 \mathrm{a}$ & $0.66 \mathrm{a}$ & $1.19 \mathrm{a}$ & $1.56 \mathrm{ab}$ & $1.68 \mathrm{a}$ & $1.89 \mathrm{~b}$ & $0.29 \mathrm{a}$ & $0.47 \mathrm{a}$ & $0.19 \mathrm{a}$ & $0.28 \mathrm{a}$ & $27.18 \mathrm{a}$ & $35.57 \mathrm{ab}$ & $31.21 \mathrm{a}$ & $26.20 \mathrm{a}$ & $48.73 \mathrm{a}$ & 68.91ab \\
\hline "Chardonnay" & $1.57 \mathrm{~b}$ & $0.70 \mathrm{a}$ & $1.26 \mathrm{ab}$ & $1.49 \mathrm{a}$ & $1.31 \mathrm{a}$ & $1.96 \mathrm{~b}$ & $0.41 \mathrm{ab}$ & $0.80 \mathrm{ab}$ & $0.26 \mathrm{ab}$ & $0.26 \mathrm{a}$ & $36.11 b$ & $36.44 b$ & $48.46 \mathrm{ab}$ & $48.52 \mathrm{a}$ & $58.61 \mathrm{a}$ & $87.00 \mathrm{~b}$ \\
\hline "Riesling" & $1.28 \mathrm{~b}$ & $0.65 \mathrm{a}$ & $1.31 \mathrm{ab}$ & $1.57 \mathrm{ab}$ & $2.56 \mathrm{~b}$ & $2.62 \mathrm{~b}$ & $0.46 b$ & $0.70 \mathrm{ab}$ & $0.37 \mathrm{~b}$ & $0.51 b$ & $41.71 \mathrm{c}$ & $43.19 \mathrm{c}$ & $54.92 \mathrm{ab}$ & $53.88 \mathrm{a}$ & $48.61 \mathrm{a}$ & $64.77 \mathrm{a}$ \\
\hline "Pinot Noir" & $1.48 \mathrm{~b}$ & $0.77 \mathrm{a}$ & $1.38 \mathrm{~b}$ & $1.62 \mathrm{ab}$ & $1.59 \mathrm{a}$ & $1.73 b$ & $0.54 \mathrm{bc}$ & $0.88 b$ & $0.19 \mathrm{a}$ & $0.24 \mathrm{a}$ & $35.68 \mathrm{~b}$ & $33.08 \mathrm{ab}$ & $76.02 b$ & $55.37 \mathrm{a}$ & $50.41 \mathrm{a}$ & $68.52 \mathrm{ab}$ \\
\hline \multicolumn{17}{|l|}{ Seasons (S) } \\
\hline 2017 & $0.87 \mathrm{a}$ & $0.60 \mathrm{a}$ & $1.41 \mathrm{~b}$ & $1.81 \mathrm{a}$ & $1.39 \mathrm{a}$ & $1.68 \mathrm{a}$ & $0.47 \mathrm{a}$ & $0.83 a$ & $0.26 \mathrm{a}$ & $0.31 \mathrm{ab}$ & $34.51 \mathrm{ab}$ & $36.70 \mathrm{a}$ & $46.58 \mathrm{a}$ & $48.92 \mathrm{a}$ & $60.86 b$ & $80.03 \mathrm{~b}$ \\
\hline 2018 & $1.10 \mathrm{a}$ & $0.68 \mathrm{a}$ & $1.23 \mathrm{a}$ & $1.53 \mathrm{a}$ & $1.87 \mathrm{a}$ & $1.95 \mathrm{a}$ & $0.46 \mathrm{a}$ & $0.74 \mathrm{a}$ & $0.32 \mathrm{a}$ & $0.44 b$ & $32.12 \mathrm{a}$ & $35.26 \mathrm{a}$ & $42.74 \mathrm{a}$ & $44.16 \mathrm{a}$ & $44.71 \mathrm{ab}$ & $65.73 \mathrm{a}$ \\
\hline 2019 & $1.70 \mathrm{~b}$ & $0.83 b$ & $1.26 \mathrm{a}$ & $1.46 \mathrm{a}$ & $1.82 \mathrm{a}$ & $1.66 \mathrm{a}$ & $0.46 \mathrm{a}$ & $0.76 \mathrm{a}$ & $0.25 \mathrm{a}$ & $0.27 \mathrm{a}$ & $35.96 \mathrm{~b}$ & $36.44 a$ & $64.76 \mathrm{a}$ & $43.23 \mathrm{a}$ & $37.49 \mathrm{a}$ & $59.54 \mathrm{a}$ \\
\hline \multicolumn{17}{|l|}{ Significance } \\
\hline Variety & 0.0133 & 0.5389 & 0.1159 & 0.0644 & 0.0336 & 0.0073 & 0.0012 & 0.0138 & 0.0059 & 0.0596 & $<0.0001$ & $<0.0001$ & 0.256 & 0.3062 & 0.28 & 0.0116 \\
\hline Season & 0.0005 & 0.0078 & 0.0096 & 0.0005 & 0.2597 & 0.6706 & 0.9886 & 0.7451 & 0.2392 & 0.0891 & 0.0442 & 0.4722 & 0.2865 & 0.8571 & 0.0793 & 0.0196 \\
\hline $\mathrm{V} \times \mathrm{S}$ & 0.1773 & 0.7985 & 0.5852 & 0.0324 & 0.856 & 0.9761 & 0.9445 & 0.9731 & 0.3361 & 0.6705 & 0.2673 & 0.0044 & 0.2737 & 0.6075 & 0.8549 & 0.2028 \\
\hline
\end{tabular}

Data are the overall mean value $(n=3$ replicates by variety). For each parameter, different letters within a column represent significant

differences (Duncan's test $\mathrm{p} \leq 0.05$ ). The numbers in red color correspond to significant difference ( $\mathrm{p}$-value lower than 0.05 ). 
The nutrient content at véraison varied between 2.1 and $2.7 \%$ for $\mathrm{N}$ ("New York Muscat" and "L'Acadie", respectively), 1.6 and $2.5 \%$ for $\mathrm{Ca}$ ("New York Muscat" and "L'Acadie", respectively), 0.8 and $1.1 \%$ for K ("L'Acadie" and "Riesling", respectively), 0.26 and $0.42 \%$ for $\mathrm{Mg}$ ("New York Muscat" and "L'Acadie", respectively), 0.23 and $0.30 \%$ for P ("New York Muscat" and "L'Acadie", respectively), 26 and $41 \mathrm{mg} / \mathrm{kg}$ for B ("L'Acadie" and "Pinot Noir" , respectively), 89 and $244 \mathrm{mg} / \mathrm{kg}$ for Fe ("New York Muscat" and "Riesling", respectively), and 25 and $120 \mathrm{mg} / \mathrm{kg}$ for $\mathrm{Zn}$ ("L'Acadie" and "New York Muscat", respectively).

$\mathrm{N}, \mathrm{Ca}, \mathrm{Mg}$ and $\mathrm{B}$ content in blades measured in bloom and véraison were significantly affected by variety (Table 4). "New York Muscat" showed lower $\mathrm{N}$ content in blades measured in both phenological stages compared to most of the studied varieties. "L'Acadie" showed the highest Ca blade content at bloom and véraison and the highest $\mathrm{Mg}$ blade content at véraison. "Chardonnay" also presented high $\mathrm{Mg}$ blade content compared to the rest of the varieties. "Pinot Noir" showed the highest B blade content at véraison, while interspecific hybrids tended to accumulate low B levels in blades at both phenological stages. Fe blade content at véraison was also affected by variety, and "Riesling" presented higher content of this micronutrient than the interspecific hybrids.

Season factor significantly affected $\mathrm{N}$ blade content analysed at véraison, and $\mathrm{Ca}$ and $\mathrm{B}$ blade content determined at bloom (Tables 2 and 4). In 2017, the grapevines presented the lowest $\mathrm{N}$ blade content at véraison and the highest $\mathrm{Ca}$ blade content at bloom. In this season, the grapevines reached higher B blade content at bloom than the samples harvested in 2018. Season and variety interaction did not affect macro and micronutrients analysed in blades at bloom and véraison stages.

Table V Nutrient blade content in bloom and véraison of interspecific hybrids and Vitis vinifera varieties cultivated in Annapolis Valley

\begin{tabular}{|c|c|c|c|c|c|c|c|c|c|c|c|c|c|c|c|c|}
\hline \multirow{2}{*}{ Variety $(V)$} & \multicolumn{2}{|c|}{$\mathbf{N}(\%)$} & \multicolumn{2}{|c|}{$\mathrm{Ca}(\%)$} & \multicolumn{2}{|c|}{$\mathbf{K}(\%)$} & \multicolumn{2}{|c|}{$\operatorname{Mg}(\%)$} & \multicolumn{2}{|c|}{$\mathbf{P}(\%)$} & \multicolumn{2}{|c|}{ B (mg/kg) } & \multicolumn{2}{|c|}{$\mathrm{Fe}(\mathrm{mg} / \mathrm{kg})$} & \multicolumn{2}{|c|}{$\mathrm{Zn}(\mathbf{m g} / \mathbf{k g})$} \\
\hline & Bloom & Véraison & Bloom & Véraison & Bloom & Véraison & Bloom & Véraison & Bloom & Véraison & Bloom & Véraison & Bloom & Véraison & Bloom & Véraison \\
\hline "L'Acadie" & $3.61 \mathrm{~b}$ & $2.73 b$ & $1.55 \mathrm{~b}$ & $2.47 \mathrm{~b}$ & $0.92 \mathrm{a}$ & $0.78 \mathrm{a}$ & $0.32 b$ & $0.42 b$ & $0.29 \mathrm{ab}$ & $0.30 \mathrm{a}$ & $29.39 a$ & $26.39 \mathrm{a}$ & $123.73 \mathrm{a}$ & $96.32 \mathrm{a}$ & $27.54 \mathrm{a}$ & $24.56 \mathrm{a}$ \\
\hline "NY Muscat" & $2.56 \mathrm{a}$ & $2.08 \mathrm{a}$ & $1.16 \mathrm{a}$ & $1.56 \mathrm{a}$ & $0.86 \mathrm{a}$ & $0.88 \mathrm{ab}$ & $0.23 \mathrm{a}$ & $0.26 \mathrm{a}$ & $0.20 \mathrm{a}$ & $0.23 \mathrm{a}$ & $23.34 \mathrm{a}$ & $28.79 \mathrm{ab}$ & $115.40 \mathrm{a}$ & $88.86 a$ & $89.46 a$ & $120.31 \mathrm{a}$ \\
\hline "Chardonnay" & $2.82 \mathrm{ab}$ & $2.73 b$ & $1.26 \mathrm{a}$ & $2.00 \mathrm{a}$ & $1.50 \mathrm{~b}$ & $0.94 \mathrm{ab}$ & $0.31 \mathrm{~b}$ & $0.31 \mathrm{a}$ & $0.34 \mathrm{~b}$ & $0.23 \mathrm{a}$ & $34.84 a b$ & $35.38 \mathrm{bc}$ & 174.19ab & $187.38 \mathrm{ab}$ & $40.90 \mathrm{a}$ & $42.82 \mathrm{a}$ \\
\hline "Riesling" & $3.52 b$ & $2.63 b$ & $1.12 \mathrm{a}$ & $1.86 \mathrm{a}$ & $1.16 \mathrm{ab}$ & $1.10 \mathrm{~b}$ & $0.24 \mathrm{a}$ & $0.33 \mathrm{a}$ & $0.25 \mathrm{ab}$ & $0.29 \mathrm{a}$ & $35.18 \mathrm{ab}$ & $39.82 \mathrm{c}$ & $261.47 b$ & $244.48 b$ & $50.00 \mathrm{a}$ & $51.52 \mathrm{a}$ \\
\hline "Pinot Noir" & $3.64 b$ & $2.71 b$ & $1.29 \mathrm{a}$ & $1.96 \mathrm{a}$ & $0.97 \mathrm{a}$ & $0.99 \mathrm{ab}$ & $0.24 \mathrm{a}$ & $0.28 \mathrm{a}$ & $0.26 a b$ & $0.27 \mathrm{a}$ & $44.19 b$ & $41.14 \mathrm{~d}$ & $152.67 \mathrm{ab}$ & $139.37 \mathrm{ab}$ & $48.30 \mathrm{a}$ & $46.03 \mathrm{a}$ \\
\hline \multicolumn{17}{|l|}{ Seasons (S) } \\
\hline 2017 & $3.09 \mathrm{a}$ & $2.31 \mathrm{a}$ & $1.46 \mathrm{~b}$ & $2.14 \mathrm{a}$ & $0.95 \mathrm{a}$ & $0.92 \mathrm{a}$ & $0.25 \mathrm{a}$ & $0.30 \mathrm{a}$ & $0.23 \mathrm{a}$ & $0.28 \mathrm{a}$ & $40.21 b$ & $34.77 \mathrm{a}$ & $203.47 \mathrm{a}$ & $162.08 \mathrm{a}$ & $82.68 b$ & $89.35 \mathrm{a}$ \\
\hline 2018 & $3.07 \mathrm{a}$ & $2.75 b$ & $1.21 \mathrm{a}$ & $1.91 \mathrm{a}$ & $1.16 \mathrm{a}$ & $0.98 \mathrm{a}$ & $0.29 \mathrm{a}$ & $0.33 \mathrm{a}$ & $0.31 \mathrm{~b}$ & $0.28 \mathrm{a}$ & $28.11 \mathrm{a}$ & $33.99 \mathrm{a}$ & $132.67 \mathrm{a}$ & $181.19 \mathrm{a}$ & $25.77 \mathrm{a}$ & $37.80 \mathrm{a}$ \\
\hline 2019 & $3.53 \mathrm{a}$ & $2.66 \mathrm{~b}$ & $1.16 \mathrm{a}$ & $1.87 \mathrm{a}$ & $1.14 \mathrm{a}$ & $0.90 \mathrm{a}$ & $0.26 \mathrm{a}$ & $0.34 \mathrm{a}$ & $0.26 \mathrm{ab}$ & $0.23 \mathrm{a}$ & $31.84 \mathrm{ab}$ & $34.16 \mathrm{a}$ & $160.34 \mathrm{a}$ & $110.58 \mathrm{a}$ & $45.26 \mathrm{ab}$ & $43.99 \mathrm{a}$ \\
\hline \multicolumn{17}{|l|}{ Significance } \\
\hline Variety & 0.0305 & 0.0005 & 0.0024 & 0.0063 & 0.0976 & 0.0556 & 0.0151 & 0.0051 & 0.0599 & 0.3935 & 0.0201 & 0.0048 & 0.0656 & 0.0325 & 0.4301 & 0.3409 \\
\hline Season & 0.2423 & 0.0011 & 0.0035 & 0.2689 & 0.4714 & 0.6415 & 0.3792 & 0.3876 & 0.0607 & 0.3109 & 0.0304 & 0.9678 & 0.2151 & 0.2052 & 0.1092 & 0.3353 \\
\hline $\mathrm{V} \times \mathrm{S}$ & 0.7086 & 0.172 & 0.8149 & 0.7476 & 0.7139 & 0.9772 & 0.852 & 0.5487 & 0.5072 & 0.8215 & 0.6469 & 0.6925 & 0.3093 & 0.9383 & 0.3221 & 0.47 \\
\hline
\end{tabular}

Data are the overall mean value $(n=3$ replicates by variety). For each parameter, different letters within a column represent significant differences (Duncan's test $p \leq 0.05$ ). The numbers in red color correspond to significant difference ( $p$-value lower than 0.05 ).

\section{Relationship between petiole versus blade nutrition status in bloom and véraison}

The coefficients of determination $\left(\mathrm{r}^{2}\right)$ for the nutrients measured in bloom was 0.97 for $\mathrm{N}, 0.98$ for $\mathrm{Ca}, 0.93$ for $\mathrm{K}, 0.95$ for $\mathrm{Mg}, 0.88$ for $\mathrm{P}, 0.97$ for $\mathrm{B}$, 0.91 for $\mathrm{Fe}$ and 0.82 for $\mathrm{Zn}$ (Figure 5), while the coefficients of determination for the nutrients measured in véraison was 0.96 for $\mathrm{N}, 0.97$ for $\mathrm{Ca}$, 0.89 for $\mathrm{K}, 0.95$ for $\mathrm{Mg}, 0.93$ for $\mathrm{P}, 0.95$ for B, 0.90 for $\mathrm{Fe}$ and 0.86 for $\mathrm{Zn}$ (Figure 5).
This three-year study shows significant nutritional imbalances in relation to the nutrient content in blades and petioles measured at bloom and véraison in the interspecific hybrids and Vitis vinifera varieties according to the nutritional references published by Jones et al. (1991). In general, these results could be conditioned by the nutritional fertility of the soil, vine age and vine yield (Tables 1 and 3). "L'Acadie" had deficiencies in N, K and $\mathrm{Mg}$ in petioles measured at véraison, while $\mathrm{B}$ levels in 
petioles were below the optimal. Low B availability is frequent in acid soils as occurs in Nova Scotia, which results in cessation of cell division (Brdar-Jokanovic, 2020). "L'Acadie" also presented $\mathrm{Ca}, \mathrm{K}$ and $\mathrm{B}$ levels below the optimal in blades and $\mathrm{N}$ and $\mathrm{Fe}$ content above the optimal in blades collected at bloom. These results are interesting since according to linear correlations bunch weight in "L'Acadie" was positively correlated with $\mathrm{K}$ contents at bloom and negatively correlated with $\mathrm{Mg}$ content in most of the samples. At high levels in soils, ammonium decrease the uptake of $\mathrm{K}, \mathrm{Ca}$ and
$\mathrm{Mg}$ and high $\mathrm{K}$ to $\mathrm{Mg}$ ratio may affect growth and Mg uptake in crops (Rayar and van Hai 1977; Li et al., 2018). $\mathrm{K}$ is implicated in enzyme activation and carbohydrate mobilization in grapes (Vasconcelos et al., 2008, Keller, 2020). K soil applications in deficient vineyards resulted in an increase in fruitfulness of latent buds in "Sultana" grapevines (Christensen, 1975). In fact, grapevines may use the soil applied $\mathrm{K}$ for growth and bud development rather than its store in the cane (Obbink et al., 1973, Vasconcelos et al., 2008).
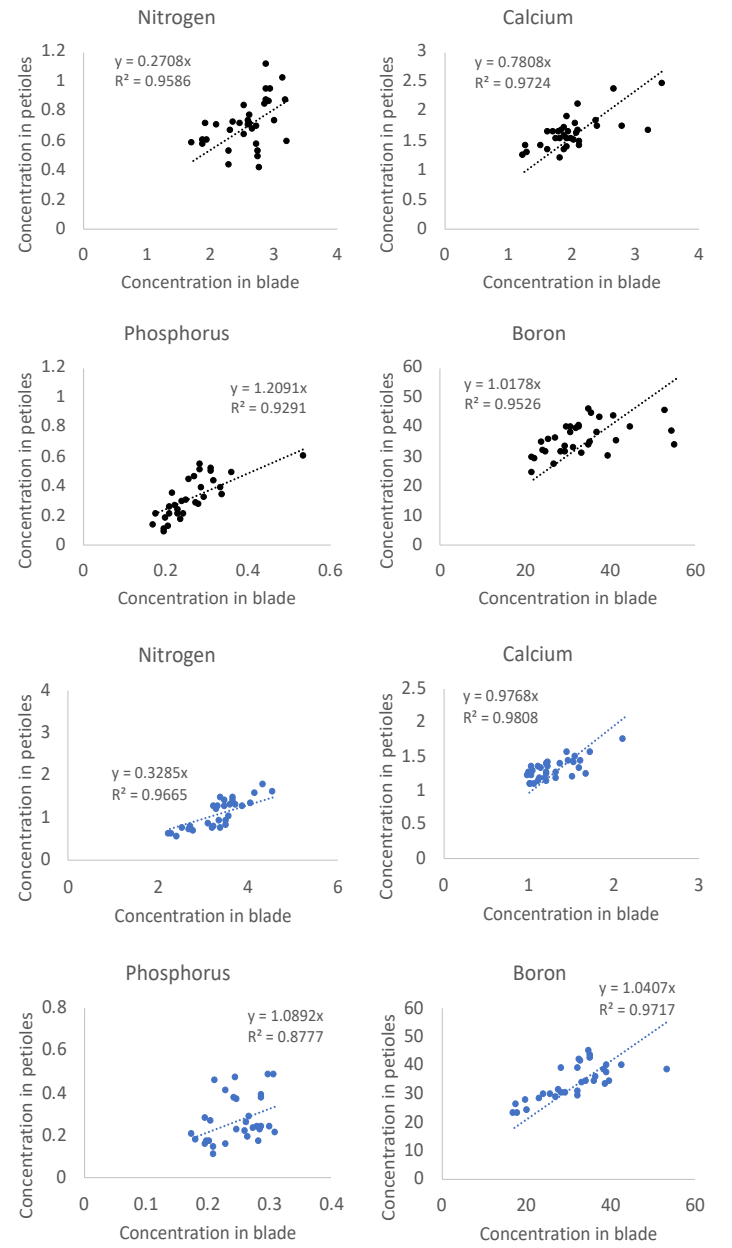
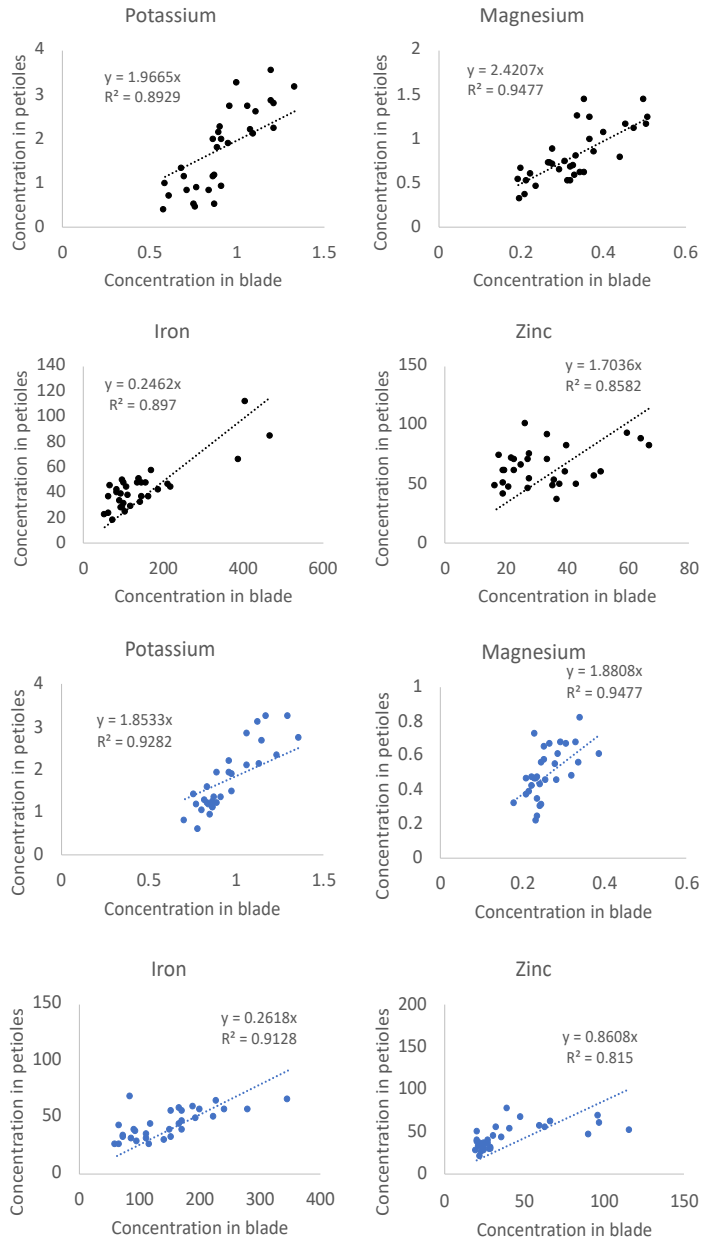

Figure 5. Relationship between petiole and blade nutrient content in bloom (in blue) and véraison (in black) in samples obtained in 2017, 2018 and 2019 seasons.

Recently, it was reported that light and $\mathrm{CO}_{2}$-saturated photosynthesis, maximum rate of ribulose 1.5 bisphospate carboxylation and maximum rate of electron transport all decreased under $\mathrm{K}$ and $\mathrm{Mg}$ deficiency (Rogiers et al., 2020). These authors also showed that $\mathrm{K}$ deficiency drastically reduced shoot biomass, while root biomass was greatly reduced under both $\mathrm{Mg}$ and $\mathrm{K}$ deficiency. It was suggested that the decrease in biomass in grapevines was likely due to both stomatal and biochemical limitations in photosynthesis under $\mathrm{Mg}$ and $\mathrm{K}$ deficiencies. This may be confirmed with yield data since "L'Acadie" presented low productivity compared to "Riesling" in most of the seasons. In addition, B petiole content analyzed in bloom was strongly correlated with yield in "L'Acadie". The grapevines require B for the germination and growth of pollen during bloom, so that deficiencies of this micronutrient will result in clusters that set numerous shot berries, small berries with a distinctive pumpkin shape (Peacock and Christensen, 2005). Indeed, berries of B-deficient grapevines had fewer and smaller seeds than B non-deficient grapevines (Hardie and Aggenbach, 1996). Most of the calculated ratios in petioles at bloom were negatively correlated with bunch 
weight, while $\mathrm{Ca}$ to $\mathrm{Mg}$ ratio calculated in petioles in bloom and véraison were correlated with plant yield. Previous studies showed that the accumulation of $\mathrm{Ca}$ is synergistic to $\mathrm{Mg}$ and that $\mathrm{Ca}+\mathrm{Mg}$ to $\mathrm{K}$ ratio was negatively correlated with yield (Muthukrishnan and Srinivasan 1974; Assimakopoulou and Tsougrianis 2012). Low $\mathrm{pH}$ in soils increase $\mathrm{K}$ availability and enhance its uptake while reducing uptake of $\mathrm{Ca}$ and Mg (Centinari, 2018). Nevertheless, as observed in most of the samples studied in this field trial, excess of $\mathrm{K}$ can decrease concentrations of $\mathrm{Ca}$ and $\mathrm{Mg}$ in plant tissues and induce $\mathrm{Mg}$ deficiencies (Keller, 2020). Seeing that, under the edaphoclimatic conditions of the Annapolis Valley, productivity in "L'Acadie" is related to $\mathrm{B}, \mathrm{K}$ and $\mathrm{Mg}$, while the accumulation of soluble solids is more related to $\mathrm{N}$ uptake.

"New York Muscat" presented deficiencies in petiolar $\mathrm{N}$ at bloom and véraison and excess of $\mathrm{Fe}$ petiole content at véraison according to the nutritional references published by Jones et al. (1991). Some nutrients in petioles analyzed at bloom such as $\mathrm{Ca}, \mathrm{Mg}, \mathrm{P}$ and $\mathrm{B}$ were in levels below the optimal nutritional values. $\mathrm{Mg}$ deficiency is a quite common predicament of grapevines growing in acidic soils (Keller, 2020). This variety also had B blade deficiencies at bloom. In addition to this, $\mathrm{Ca}$ and $\mathrm{K}$ blade content was below to the optimal, while $\mathrm{Fe}$ and $\mathrm{Zn}$ blade content was above the optimal nutritional values. "New York Muscat" soil analysis revealed lower $\mathrm{CaO}$ and higher $\mathrm{Zn}$ content than the rest of the vineyard soils (Table 3). Deficiencies in nutrients and toxicity by metals, such as $\mathrm{Mn}, \mathrm{Zn}, \mathrm{Fe}$ and $\mathrm{Al}$ are characteristics of acid soils (Joshi et al., 2014; Bojórquez-Quintal et al., 2017). Fe toxicity is rare under natural vineyard conditions and is mainly limited to extreme acid soils (Keller, 2020). In "New York Muscat", bunch weight was positively correlated with $\mathrm{N}$ levels at véraison and negatively correlated with $\mathrm{Ca}$ to $\mathrm{K}$. $\mathrm{N}$ in adequate levels are necessary for inflorescence primordium formation and for differentiation of flowers (Vasconcelos et al., 2008). Contrary to our results, bunch weight was not affected by foliar applications to grapevines at véraison (Tozzini et al., 2013). Low N status may be associated with berry growth (Hilbert et al., 2003; Rogiers et al., 2004). Similar to our results, it was reported that in "Italia" ("Bicane" $\times$ "Muscat Hamburg") grapevines, the decrease of $\mathrm{N}$ content in the primary leaves after flowering indicated a remobilization toward the clusters, which acted as a stronger sink (Ferrara et al., 2018). As "Italia", "New York Muscat" ("Muscat Hamburg" $\times$ "Ontario") is a vigorous and woody grapevine that is characterized by clusters of edibles, juicy, sweet, dark maroon to blue-black fruit. $\mathrm{K}$ to $\mathrm{Mg}$ ratio calculated in blades at bloom was positively correlated with weight of berries in "New York Muscat" grapevines. K and Mg provide as cation in the regulation of the cation-anion balance and as osmotically active ion in turgor regulation of cells (Gerendás and Führs, 2013). Zn content analysed in bloom was positively correlated with yield in this variety. As B, insufficient $\mathrm{Zn}$ supply can inhibit pollen formation and, therefore, pollination, leading to poor fruit set (Keller, 2020). Owing to this, "New York Muscat" productivity is related to $\mathrm{Zn}$, while $\mathrm{N}, \mathrm{P}$ and $\mathrm{K}$ may affect its yield components. "New York Muscat" is characterized by possessing large berries with a great weight, which could condition the nutritional deficiencies presented in the results. Based upon the ratios calculated it is possible that "New York Muscat" grapevines growing under the edaphoclimatic conditions of Nova Scotia may react positively to $\mathrm{K}$ soil applications.

"Chardonnay" presented excessive petiole levels of $\mathrm{N}$ at bloom and deficient $\mathrm{N}$ levels at véraison according to the nutritional values published by Jones et al. (1991). Ca and K measured in petioles at bloom was below to the optimal nutrient values in this variety, while $\mathrm{Zn}$ petiole level reached values above the optimal. $\mathrm{N}$ and $\mathrm{P}$ levels in blades measured in bloom as well as $\mathrm{Fe}$ and $\mathrm{Zn}$ blade levels were above the optimal nutritional values in "Chardonnay", while Ca measured at bloom and $\mathrm{K}$ determined at véraison in "Chardonnay" blades were below to the optimal nutritional. In this variety, Fe petiole content determined at véraison was positively correlated with bunch weight and weight of berries but negatively correlated with total acidity and yeast assimilable nitrogen. In general, "Chardonnay" grapevines established in Nova Scotia produce bunch with high weight, which may condition the nutritional unbalances presented in the results. Fe is involved in chlorophyll synthesis, photosynthesis, and respiration and it is also implicated in $\mathrm{C}, \mathrm{N}$, and $S$ assimilation via enzyme activation (Keller, 2020) $\mathrm{Fe}$ is involved in lipid and hormone synthesis and degradation, and DNA synthesis and repair, as well as detoxification of reactive oxygen species (Keller, 2020). Fe is also abundant in seeds and pollen and may be necessary for pollen production (Curie et al., 2009). The decrease of photosynthesis in grapevines affected by strong iron deficiency led to a reduction in accumulation of substances in the berries during ripening, increasing total acidity (Martín et al., 2008; González et al., 2019).

The generally observed low $\mathrm{Fe}$ content in the grapevines was in contrast with the revealed high $\mathrm{Fe}$ availability in the soil (data not shown). Fe may be abundant in calcareous soils but, it is often precipitated as insoluble $\mathrm{Fe}^{3+}$ oxides and hydroxides that results in an unavailable of Fe by roots (Keller 2020). Bicarbonate uptake from calcareous soils inhibit the conversion of the inactive form $\left(\mathrm{Fe}^{3+}\right)$ to the active form of iron $\left(\mathrm{Fe}^{2+}\right)$, and $\mathrm{Fe}$ becomes "locked up" in the apoplast and cannot enter the mesophyll cells (Keller, 2020). Thus, Fe may also accumulate in the roots (Gruber and Kosegarten 2002; Keller, 2020). As stated previously, this "iron chlorosis paradox" is frequent in scion varieties grafted onto Vitis riparia rootstocks grown on 
high-pH soils (Gruber and Kosegarten, 2002). In our study, $3309 \mathrm{C}$ was the rootstock selected for the $V$. vinifera varieties by winegrowers and has a low tolerance to total limestone (20\%), active limestone $(11 \%)$ and accounts an IPC of 10 . Therefore, under the edaphoclimatic conditions of Nova Scotia viticulture.

"Riesling" had deficiencies in $\mathrm{N}$ at véraison and excess in $\mathrm{P}$ at véraison in petioles according to the nutritional values published by Jones et al. (1991). This variety showed $\mathrm{K}$ petiole levels above the optimal nutritional values and the highest levels of this macronutrient measured at bloom. These results could have been conditioned by yield, since in the first and second seasons "Riesling" grapevines presented the highest yield per vine. Moreover, "Riesling" grapevines were stablished between 2013 and 2015 after the other varieties, which could also influence the $\mathrm{K}$ content in leaf tissues. "Riesling" reached excess Fe levels in blades at bloom, while $\mathrm{Zn}$ levels in blades were above the optimal nutritional values. Soil nutritional analysis reported that "Riesling" grapevines are growing under high $\mathrm{Fe}$ and $\mathrm{Zn}$ conditions (Table 3). For its part, $\mathrm{Ca}$ in blades presented levels below the nutritional optimum in this variety. "Riesling" is a Vitis vinifera variety that has been well received by vine growers in the Annapolis Valley due to its great oenological potential and its high level of bud cold hardiness regarding $V$. vinifera varieties. However, $\mathrm{K}$ and yield management has become an important topic to follow in vineyards grown under cold weather conditions mostly due to excesses rather than deficiencies of this macronutrient. Grapes are a strong sink for $\mathrm{K}$ during grape ripening and it is accumulated mainly in berry skin, representing the main cation found in musts (Rogiers et al., 2006). High levels of $\mathrm{K}$ in musts may result in high must $\mathrm{pH}$ reducing wine stability (Mpelasola et al., 2003). However, low $\mathrm{pH}$ wines may also have high $\mathrm{K}$ levels (Centinari, 2018). During wine making, high concentration of $\mathrm{K}$ causes precipitation of free acids, mainly tartaric acid, leading to an increased wine $\mathrm{pH}$ (Mpelasoka et al., 2003, Centinari, 2018). Grapes contains significant amounts of tartaric acid and K, such that musts contain a high content of potassium bitartrate (KHT) (Coulter et al., 2015). The formation of crystalline deposits is a phenomenon of wine aging related to KHT which in general is not meet with consumer acceptance (Bosso et al., 2020). Bunch weight was positively correlated with $\mathrm{Ca}$ to $\mathrm{Mg}$ ratio calculated in blades at véraison and negatively related to $\mathrm{Mg}$ blade content measured at this same phenological stage. Since $\mathrm{Mg}$ is capable of replacing $\mathrm{Ca}$ from binding sites, the imbalance in $\mathrm{Ca}$ to $\mathrm{Mg}$ ratio in plant tissues often negatively affect product quality (Gerendás and Führs, 2013).

Therefore, yield and K management in "Riesling" grapevines by potential $\mathrm{K}$ excesses and in the rest of the varieties by deficiencies should be considered a priority in the nutritional management program under the edaphoclimatic conditions of the Annapolis Valley. The generalized low to very low grapevine $\mathrm{K}$ content during the whole vegetative period in most of the grapevine varieties established in Nova Scotia and the good soil $\mathrm{Mg}$ availability makes $\mathrm{K}$ deficiency more important (Grallert and Laytte, 2018). Given the importance of $\mathrm{K}$ in the grapevine grape development process, regular $\mathrm{K}$ soil amendments and, additionally, sulfate foliar applications on the canopy are recommended for grapevines cultivated in Nova Scotia (Grallert and Laytte, 2018).

"Pinot Noir" presented excessive levels of $\mathrm{N}$ at bloom and deficient $\mathrm{N}$ levels at véraison in petioles according to the nutritional values published by Jones et al. (1991). Ca and P petiole content at bloom reached levels below to the nutritional optimum, while $\mathrm{Mg}$ at véraison and $\mathrm{Fe}$ content in petioles were above the optimum values. $\mathrm{N}$ in blades at bloom, $\mathrm{P}$ in blades at véraison together with $\mathrm{Fe}$ and $\mathrm{Zn}$ in blades reached values below to the nutritional optimum, while $\mathrm{Ca}$ and $\mathrm{P}$ showed the opposite behavior. "Pinot Noir" showed the highest B blade content in véraison. In this variety, soluble solids were positively correlated with $\mathrm{Mg}$ and negatively with $\mathrm{K}$ petiole content determined at bloom and $\mathrm{Ca}$ to $\mathrm{Mg}$ ratio calculated in petioles at véraison. $\mathrm{Mg}$ deficiency is a typical problem of acidic soils (Gransee and Führs, 2013). Soluble solids and acid concentration in fruits increased after the dolomitic limestone application for seven years in acidic and Mg-deficient soils (Quaggio et al., 1992). These increases were strongly correlated with the leaf $\mathrm{Mg}$ concentrations. In addition, it was suggested that fruit acidity might be related to an increase in $\mathrm{Ca}$ to $\mathrm{Mg}$ ratio rather than the increase in $\mathrm{K}$ to $\mathrm{Mg}$ ratio (Moss and Higgins, 1974). It is possible that the high acidity found in fruits after a high $\mathrm{Mg}$ uptake without changes in $\mathrm{K}$ absorption may results into increase in organic acids to counterbalance the increased cellular cation accumulation (Gransee and Führs, 2013). "Pinot Noir" yield was positively correlated to $\mathrm{K}$ to $\mathrm{Ca}$ ratio calculated in blades at véraison. $\mathrm{K}$ play a key role in cellular growth and cell walls in the berry skin loosens at the onset of stage III of berry growth (Mpelasoka et al., 2003). The cell walls loosen involves the apoplast acidification and the activation of cell wall loosening enzymes (Raath, 2012). Kirkby and Pilbeam (1984) showed that the fruits that grow fast have a higher $\mathrm{K}$ to $\mathrm{Ca}$ ratio than the slower growing fruit. Rogiers et al. (2006) indicated that due to a strong correlation found between $\mathrm{K}$ accumulation and berry fresh mass, $\mathrm{K}$ plays a key role in cell expansion, and therefore in berry growth. Seeing that, $\mathrm{K}$ to $\mathrm{Ca}$ ratio could be an important indicator of yield in "Pinot Noir" variety.

The most reliable analytical tool that provides a guide for vineyard nutrient management are the tissues analysis which is more effective than soil analysis (Christensen, 1969; Assimakopoulou and Tsougrianis, 2012). Taking into account the 
nutritional differences among varieties, interspecific hybrids accumulate lower B content than the Vitis vinifera varieties. "L'Acadie" tended to accumulate high amounts of $\mathrm{Ca}$ and $\mathrm{Mg}$, while "New York Muscat" had lower content of $\mathrm{N}$. The petiole analysis at bloom phenological stage in grapevines is considered to be a preferable tissue determination for $\mathrm{N}$ and $\mathrm{P}$ diagnosis whereas $\mathrm{K}$ measurements at this stage were found to be inadequate according to the exposed by Hepner and Bravdo (1985). Conversely, Christensen (1984) stated that the K status of the grapevine is better reflected by petioles than by blade contents. For vineyards cultivated under cold-climate conditions is suggested that the preferred time for leaf petiole testing is bloom and late-summer (70 to 100 days after bloom), and in case of suspected K or other nutrient deficiency, the samples can be collected anytime during the season (Centinari, 2018). Derived from our results, coefficients of determination $\left(\mathrm{r}^{2}\right)$ for the nutrients measured in bloom was higher than 0.82 , while for the nutrients measured in véraison was higher than 0.86. The high correlation between the petiole and leaf analysis would allow to producers to perform a single analysis to determine the grapevine nutritional status being able to save money and time.

\section{CONCLUSIONS}

Seeing our results, nutritional status of the varieties differentially affected yield, yield components and must parameters under the edaphoclimatic conditions of the Annapolis Valley. However, the number of data available in this study is very small and it will be necessary to continue this study by extending the observations to more plots occupied with the same varieties. Cold weather conditions, especially the great frost that occurred in 2018, strongly influenced the results of this field trial. Based to our results, "L'Acadie" yield was related to $\mathrm{B}, \mathrm{K}$ and $\mathrm{Mg}$, while the accumulation of soluble solids in this variety was more related to $\mathrm{N}$ uptake. Despite these results, it is of wide importance to perform optimal pruning and fertilization management and a correct use of cover crops, which can also affect yield and soluble solids accumulation in this variety. "L'Acadie" tended to accumulate high levels of Ca and Mg. "New York Muscat" yield was strongly affected by Zn, while N, P and K affects considerably bunch weight and weight of berries. "New York Muscat" also tended to accumulate low levels of N. Both interspecific hybrids presented low accumulation of B in their tissues. However, to date, it is not aware of the microelement deficiency values in hybrid varieties, but this value should be lower than the $V$. vinifera varieties. "New York Muscat" presents natural reproductive problems that results in berries with different sizes. This trouble may not only be affected by the petiole B content in bloom, but also by pollen morphology of the grapevine. "Chardonnay" yield and grape quality was related to
$\mathrm{Zn}$ and Fe contents, while "Riesling" showed the highest levels of $\mathrm{K}$ measured at bloom and its amounts on petiole were above the optimal nutritional values recommended for grapevines. Thereby, K management should be considered a priority in the nutritional management program by vine growers of the Annapolis Valley. In "Pinot Noir", K to Ca ratio could be an important indicator of yield. Coefficients of determination $\left(\mathrm{r}^{2}\right)$ for the nutrients measured in petioles and blades in véraison and bloom were higher than 0.82 .

Overall, the problems observed in relation to nutritional management in the vineyards of Nova Scotia are lack of $\mathrm{Fe}, \mathrm{K}$ and excess of $\mathrm{Mg}$ and $\mathrm{Mn}$. Therefore, the correction of the soil $\mathrm{pH}$ is crucial by the use of calcitic limestone rather than dolomitic limestone. Despite this, the results obtained are nothing more than a first contribution or approximation to achieve the proposed nutritional management objectives.

\section{ACKNOWLEDGEMENTS}

The authors would like to acknowledge Nova Scotia Department of Agriculture (NSDA) for supporting this study, grape growers of Nova Scotia for their incredible collaboration, and AAFC Kentville for their help. In terms of analysis, the authors would like to acknowledge the provincial lab for all the tissues analysis and the soil analysis to Aurea AgroSciences. Finally, the authors would like to thank the colleagues at Perennia, especially Allison Gutknecht-Segovia, Jill MacDonald, Caitlin Congdon, Myles Mcilveen and Jonathan Bent.

\section{REFERENCES}

AOAC International., 2005. Official Methods of Analysis of AOAC International 18th Edition, 2005. 4.8.02 AOAC Official Method 968.08, modified method using ICP-OES

AOAC International., 2006. Official Methods of Analysis of AOAC International 18th Edition, 2005. 4.2.08 AOAC Official Method 990.03, Combustion Method.

Assimakopoulou A., Tsougrianis C., 2012. Correlation between yield, must attributes and nutritional status of the greek red wine grape variety "Agiorgitiko." J. Plant Nut. 35, 1022-1036.

Bishop R.F., MacEachern C.R., MacLeod L.B., Jackson L. P., 1969. Effect of surface-applied limestone and superphosphate on herbage production and on some chemical properties of a Dykeland soil. Can. J. Soil Sci. 49, 47-51.

Bojórquez-Quintal E., Escalante-Magaña C., Echevarría-Machado I., Martínez-Estévez M., 2017. Aluminum, a friend or foe of higher plants in acid soils. Front. Plant Sci. 8, 1767.

Bosso A., Motta, S., Panero L., Petrozziello M., Asproudi A., Lopez R., Guaita M., 2020. Use of polyaspartates for the tartaric stabilisation of white and red wines and side effects on wine characteristics. OENO One. 54, 15-26.

Brdar-Jokanović M., 2020. Boron toxicity and deficiency in agricultural plants. Int. J. Mol. Sci. 21, 1424. 
Cameron B.I., Ketter B.S., Karakis S., 2012. An emerging wine region in Nova Scotia, Canada: Terroir trials and tribulations. Am.n Geophys. Union 53,1590.

Centinari M., 2018. Assessing and managing potassium concentration in the vineyard. PennState Extension. Available at:

https://extension.psu.edu/assessing-and-managing-potassium -concentration-in-the-vineyard (accessed on 06.05.2021).

Christensen P., 1969. Seasonal changes and distribution of nutritional elements in Thomson seedless grapevines.

Christensen P., 1975. Long-term responses of 'Thompson Seedless' vines to potassium fertilizer treatment. Am. J. Enol. Vitic. 26, 179-183.

Christensen P., 1984. Nutrient level comparisons of leaf petioles and blades in twenty-six grape cultivars over three years (1979 through 1981). Am. J. Enol. Vitic. 35, 124-133.

Coulter A.D., Holdstock, M.G., Cowey, G.D., Simos C.A., Smith P.A., Wilkes E.N., 2015. Potassium bitartrate crystallisation in wine and its inhibition. Aust. J. Grape Wine Res. 21, 627-641.

Curie C., Cassin G., Couch D., Divol F., Higuchi K., Le Jean M., Misson J., Schikora A., Czernic P., Mari S., 2008. Metal movement within the plant. contribution of nicotianamine and yellow stripe 1-like transporters. Annals of Botany, 103(1), $1-11$

Fageria N.K., Nascente A. S., 2014. Management of soil acidity of South American soils for sustainable crop production. Adv. Agron. 128, 221-275.

Ferrara G., Malerba A.D., Matarrese A.M.S., Mondelli D., Mazzeo A., 2018. Nitrogen distribution in annual growth of "Italia" table grape vines. Front. Plant Sci. 9, 1374.

Fisher K.H., Jamieson A.R., 2000. L'Acadie, a cold hardy, white wine grape cultivar for low heat unit regions. Acta Hortic. 528, 563-567.

Gerendás J., Führs H., 2013. The significance of magnesium for crop quality. Plant Soil. 368, 101-128.

González M.R., Hailemichael G., Catalina Á., Martín P., 2019. Combined effects of water status and iron deficiency chlorosis on grape composition in non-irrigated vineyards. Sci. Agric. 76, 473-480.

Grallert C., Laytte R., 2018. Executive summary. Terroir analysis for the Nova Scotia wine growing region. Nova Scotia Department of Agriculture (NSDA). Available at: https://www.perennia.ca/wp-content/uploads/2018/03/Terroi r-Report-Executive-Summary.pdf

Grallert C., Laytte R., 2020. Executive summary. Terroir analysis for the Nova Scotia wine growing region. Nova Scotia Department of Agriculture (NSDA). Available at: https://www.perennia.ca/wp-content/uploads/2020/04/2019Report-Executive-Summary.-Perennia.pdf

Gransee A., Führs H., 2012. Magnesium mobility in soils as a challenge for soil and plant analysis, magnesium fertilization and root uptake under adverse growth conditions. Plant and Soil, 368, 5-21.

Gruber B., Kosegarten H., 2002. Depressed growth of non-chlorotic vine grown in calcareous soil is an iron deficiency symptom prior to leaf chlorosis. J. Plant Nutr. Soil Sci. 165, 111-117.

Gupta U.C., Calder F.W., Macleod L. B., 1971. Influence of added limestone and fertilizers upon the micro-nutrient content of forage tissue and soil. Plant Soil. 35(1-3), 249-256.

Hardie W.J., Aggenbach S.J., 1996. Effects of site, season and viticultural practices on grape seed development. Aust. J. Grape Wine Res. 2, 1-4.

Hepner Y., Bravdo B., 1985. Effect of crop level and drip irrigation scheduling on the potassium status of Cabernet
Sauvingon and Carignane vines and its influence on must and wine composition and quality. Am. J. Enol. Vitic. 36, 140-147.

Hilbert G., Soyer J.P., Molot C., Giraudon J., Milin S., Gaudillère, J.P., 2003. Effects of nitrogen supply on must quality and anthocyanins accumulation in berries of $\mathrm{cv}$. Merlot. Vitis. 42, 69-76.

Huglin P., 1978. Nouveau mode d'évaluation des possibilités héliothermiques d'un milieu viticole. Compt. Ren. Acad. Agric. Fr. 64, 1117-1126.

Jensen K.I.N., Doohan D.J., Specht E.G., 2004. Response of processing carrot to metribuzin on mineral soils in Nova Scotia. Can. J. Soil Sci. 84, 669-676.

Jones J.B., Wolf J.R.B., Mills H.A., 1991. Plant Analysis Handbook. Athens, GA. Micro Macro Publishing, Inc.

Joshi D., Chandra Srivastava P., Dwivedi R., Pratap Pachauri S., 2014. Chemical speciation of $\mathrm{Zn}$ in acidic soils. suitable soil extractant for assessing $\mathrm{Zn}$ availability to maize (Zea mays L.). Chem. Speciation Bioavailability. 26, 148-157.

Jones G.V., 2005. Climate and terroir. Impacts of climate variability and change on wine. Geosci. Can. 1-14.

Keller M., 2020. The science of grapevines. Anatomy and Physiology. 3rd Edition. Elsevier. Academic Press.

Kirkby E.A., Pilbeam D.J., 1984. Calcium as a plant nutrient. Plant, Cell Environ. 7, 397-405.

Li H., Chen Z., Zhou T., Liu Y., Zhou J., 2018. High potassium to magnesium ratio affected the growth and magnesium uptake of three tomato (Solanum lycopersicum L.) cultivars. J. Integr. Agric. 17, 2813-2821.

Martín P., Zarco-Tejada P.J., González M.R., 2008. Nutritional diagnosis and fertilizer recommendations in the limestone soils of Ribera de Duero. Vida Rural. 270, 26-32.

Messiga A. J., Gallant K. S., Sharifi M., Hammermeister A., Fuller K., Tango M., Fillmore S., 2015. Grape yield and quality response to cover crops and amendments in a Vineyard in Nova Scotia, Canada. Am. J. Enol. Vitic. 67, 7785 .

Moss G.I., Higgins M.L., 1974. Magnesium influences on the fruit quality of sweet orange (Citrus sinensis L. Osbeck). Plant Soil. 41, 103-112.

Mpelasoka B.S., Schachtman D.P., Treeby M.T., Thomas M.R., 2003. A review of potassium nutrition in grapevines with special emphasis on berry accumulation. Aust. J. Grape Wine Res. 9, 154-168.

Muthukrishnan C.E., Srinivasan C., 1974. Correlation between yield, quality, and petiole nutrients in grapes. Vitis. 12(4), 277-285.

Naugler C., McCallum J.L., Klassen G., Strommer J., 2007. Concentrations of trans-resveratrol and related stilbenes in Nova Scotia wines. Am. J. Enol. Vitic. 58, 117-119.

Neina D., 2019. The role of soil pH in plant nutrition and soil remediation. Appl. Environ. Soil Sci. 5794869, 1-9.

Obbink J.G., Alexander D.M.E., Possingham J.B., 1973. Use of nitrogen and potassium reserves during growth of grape vine cuttings. Vitis. 12, 207-213.

OIV., 2003. Compendium of international methods of wine and must analysis. OIV, Paris.

OIV., 2009. Compendium of international methods of wine and must analysis. Organic acids. Method OIV-MA-AS313-04. OIV, Paris.

Peacock W.L., Christensen L.P., 2005. Drip irrigation can effectively apply boron to San Joaquin Valley vineyards. Calif. Agric. 59, 188-191. 
Pedneault K., Provost C., 2016. Fungus resistant grape varieties as a suitable alternative for organic wine production. Benefits, limits, and challenges. Sci. Hortic. 208, 57-77.

Point E., Tyedmers P., Naugler C., 2012. Life cycle environmental impacts of wine production and consumption in Nova Scotia, Canada. J. Clean. Prod. 27, 11-20.

Quaggio J.A., Sobrinho J.T., Dechen A.R., 1992. Magnesium influences on fruit yield and quality of 'Valencia' sweet orange on Rangpur lime. Proc. Int. Soc. Citric. 2, 633-637.

Raath P.J., 2012. Effect of varying levels of nitrogen, potassium and calcium nutrition on table grape vine physiology and berry quality. Doctoral Thesis. Department of Viticulture and Oenology, Faculty of AgriSciences. Stellenbosch University.

Rayar A.J., van Hai T., 1977. Effect of ammonium on uptake of phosphorus, potassium, calcium and magnesium by intact soybean plants. Plant Soil. 48, 81-87.

Rengel Z., 2015. Availability of $\mathrm{Mn}, \mathrm{Zn}$ and Fe in the rhizosphere. J. Soil Sci. Plant Nutr. 15, 397-409.

Rogiers S.Y., Greer D.H., Hatfield J.M., Orchard B.A., Keller M., 2006. Mineral sinks within ripening grape berries (Vitis vinifera $\mathrm{L}$.). Vitis. 45, 115-123.

Rogiers S.Y., Greer D.H., Moroni F.J., Baby T., 2020. Potassium and magnesium mediate the light and $\mathrm{CO}_{2}$ photosynthetic responses of grapevines. Biology. 9, 144.

Rogiers S.Y., Hatfield J.M., Keller M., 2004. Irrigation, nitrogen, and rootstock effects on volume loss of berries from potted Shiraz vines. Vitis. 43, 1-6.

Rutkowska B., Szulc W., Spychaj-Fabisiak E., Pior N., 2017. Prediction of molybdenum availability to plants in differentiated soil conditions. Plant Soil Environment. 63, 491-497.

Sangster A., 2018. Nova Scotia Soil. Fact Sheet. Perennia Food and Agriculture Inc. Available at: http.//nsnewfarmer.ca/wp-content/uploads/sites/5/2018/02/N ova-Scotia-Soil.pdf

Shaw A. B., 2007. The emerging cool climate wine regions of eastern Canada. J. Wine Res. 10, 79-94.

Skoutelas D., Ricardo-da-Silva J., Laureano O., 2011. Validation and comparison of formol and FT-IR methods for assimilable nitrogen in vine grapes. S. Afr. J. Enol. Vitic. 32, 262-266.

Tonietto J., Carbonneau A., 2004. A multicriteria climatic classification system for grape-growing regions worldwide. Agric. For. Meteorol. 124, 81-97.

Tozzini L., Sabbatini P., Howell G.S., 2013. Increasing nitrogen availability at veraison through foliar applications. Implications for leaf assimilation and fruit ripening under source limitation in 'Chardonnay' (Vitis vinifera L.) grapevines. HortScience. 48, 608-613.

Vasconcelos M.C., Greven M., Winefield C.S., Trought M.C.T., Raw V., 2008. The flowering process of Vitis vinifera: A review. Am. J. Enol. Vitic. 60, 411-434.

Winkler A.J., 1974. General viticulture. $2^{\circ}$ Edition. University of California Berkeley.

Withers P., 2015. Nova Scotia offers \$1M incentive to double grape production. Canadian Broadcasting Corporation. Available

https.//www.cbc.ca/news/canada/nova-scotia/nova-scotia-wi ne-boost-1.3355478 (accessed on 06.05.2021) 